Research Article

\title{
Systems Biology and Chemoinformatics-Based Strategies to Explore the Biological Mechanism of Fugui Wenyang Decoction in Treating Vascular Dementia Rats
}

\author{
Kailin Yang $\mathbb{D},{ }^{1,2}$ Liuting Zeng $\mathbb{D}^{1},{ }^{1}$ Anqi Ge, ${ }^{2}$ Chuandong Cao, ${ }^{1}$ Haiyan Zhang, ${ }^{1}$ \\ Tingting Bao, ${ }^{3}$ Yaqiao Yi, ${ }^{1}$ and Jinwen Ge ${ }^{1}{ }^{1}$ \\ ${ }^{1}$ Hunan University of Chinese Medicine, Changsha, Hunan Province, China \\ ${ }^{2}$ The First Affiliated Hospital of Hunan University of Chinese Medicine, Changsha, Hunan Province, China \\ ${ }^{3}$ Beijing University of Chinese Medicine, Beijing, China \\ Correspondence should be addressed to Jinwen Ge; 001267@hnucm.edu.cn
}

Received 14 January 2021; Accepted 24 August 2021; Published 7 October 2021

Academic Editor: Jos P. Andrade

Copyright (c) 2021 Kailin Yang et al. This is an open access article distributed under the Creative Commons Attribution License, which permits unrestricted use, distribution, and reproduction in any medium, provided the original work is properly cited.

\begin{abstract}
Objective. To explore the biological mechanism of Fugui Wenyang Decoction (FGWYD) in treating vascular dementia (VD) rats based on systems pharmacology, proteomics, and a multidirectional pharmacology integration strategy. Methods. Chemoinformatics was utilized to construct and analyze the FGWYD-VD protein-protein interaction (PPI) network. Then, the total protein in the brain tissue of the infarcted side of the rat was extracted for protein identification, pattern identification, and protein quantitative analysis. The differentially expressed proteins are analyzed by bioinformatics. Finally, the important proteins in the oxidative stress-related biological process proteins and indicators were detected through experimental pharmacology to verify the findings of systems biology and chemoinformatics. Results. There were a total of 73 FGWYD components with 245 FGWYD and 145 VD genes. The results of GO enrichment analysis and pathway enrichment analysis showed that MBHD may regulate the inflammation module, oxidative stress, the synaptic plasticity regulation module, and the neuronal apoptosis section module. Compared with the sham operation group, there were 23 upregulated proteins and 17 downregulated proteins in the model group $(P<0.05)$. Compared with the model group, there were 16 upregulated proteins and 10 downregulated proteins in the FGWYD group $(P<0.05)$. Bioinformatics analysis shows that those proteins were closely related to processes such as inflammation, oxidative stress, neuronal apoptosis, neuronal growth and differentiation, signaling pathways, and transcriptional regulation. Multidirectional pharmacology further verified the neuroprotective mechanism of the Nrf2/HO-1 pathway in FGWYD treatment of VD. Conclusion. The mechanism of FGWYD in the treatment of VD may be related to inflammation, oxidative stress, angiogenesis, and neuronal apoptosis.
\end{abstract}

\section{Introduction}

Vascular dementia (VD) refers to a clinical syndrome characterized by a decline in cognitive functions such as learning ability, memory function, computing power, and orientation caused by various cerebrovascular accidents $[1,2]$. The incidence of VD accounts for about $20 \%$ of all dementia types [3]. Epidemiological studies have shown that with the extension of lifespan and the increase in the incidence of cerebrovascular diseases, the population affected by VD is also increasing year by year. It is predicted that by 2040 , the number of people with dementia worldwide will reach 81.1 million [4]. VD is divided into four categories according to the causes and clinical manifestations [5]: (1) mild vascular cognitive impairment, (2) mixed dementia, (3) dementia caused by vascular disease, and (4) postapoplectic dementia. The clinical manifestations of VD are related to the location, size, and number of infarctions. The symptoms of patients can be roughly divided into memory loss, abnormal executive function, daily activity ability, and mental and behavioral 


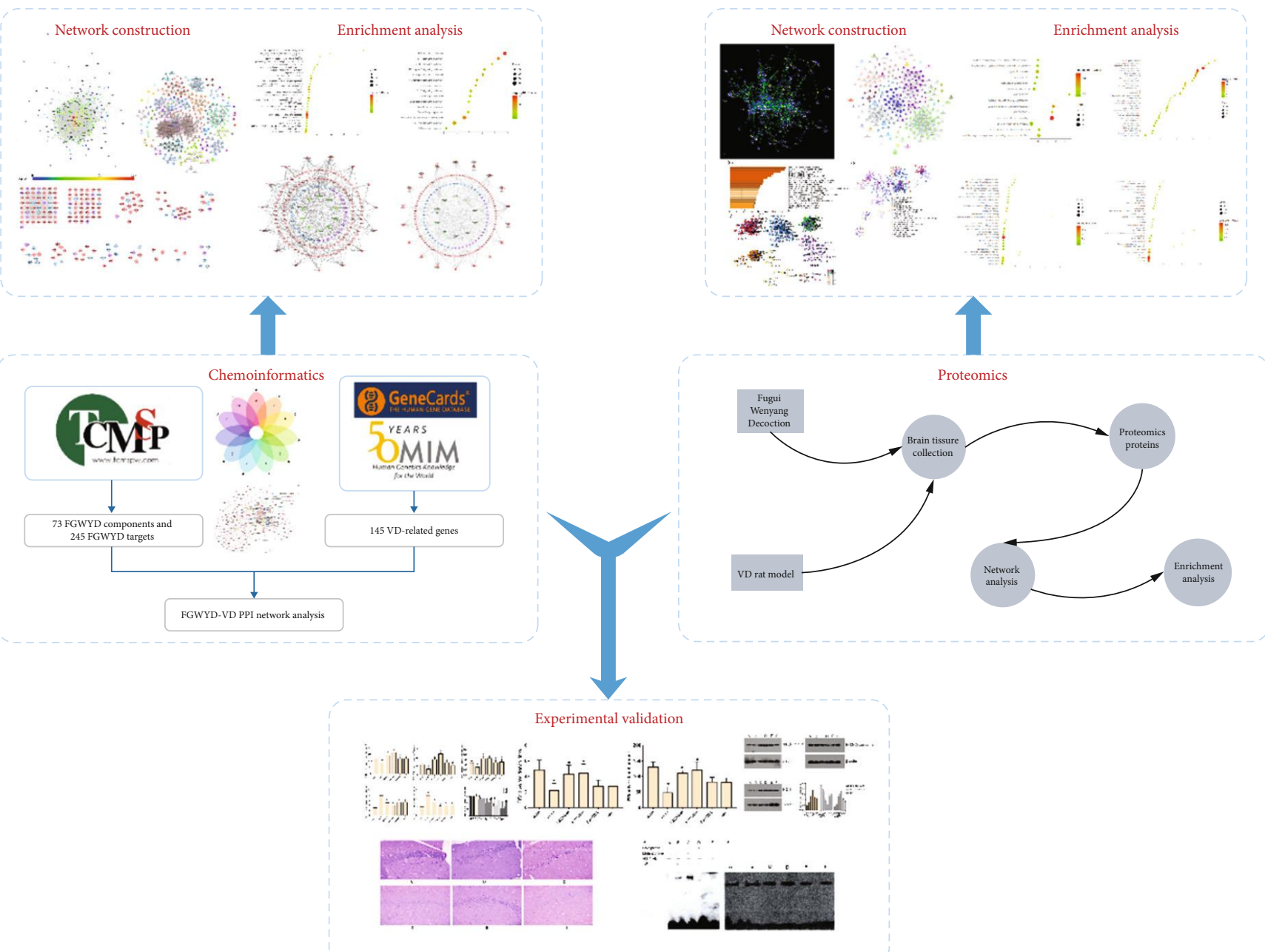

Figure 1: Research ideas.

abnormalities. The pathogenesis of VD has not been fully elucidated, and it may be closely related to excitatory amino acid toxicity, nerve cell apoptosis, free radical damage, and inflammatory response [6-8]. Neuroimaging and pathological studies have confirmed that due to cerebral vascular obstruction or traumatic lesions, insufficient cerebral blood flow perfusion is the main factor for cognitive dysfunction caused by VD [8].

At present, the drugs used in the treatment of VD are roughly classified into the following categories [9-11]: (1) acetylcholinesterase inhibitors, such as donepezil and galantamine; (2) brain circulation- and brain metabolismpromoting drugs, such as piracetam, pyrithione, and ergot alkaloid; and (3) neuroprotective drugs, such as calcium antagonists and antioxidants. Clinically, VD often requires a combination of these drugs to promote VD management. Traditional Chinese Medicine (TCM) has played an active role in VD management. TCM has a significant impact on the clinical prevention and treatment of VD because of its synergistic combination and fewer side effects. For singletarget chemical drugs, the application of the compound effectively compensates for the defects of Western medicine in clinical application, and has great advantages $[12,13]$.
Our team created Fugui Wenyang Decoction (FGWYD) based on a large number of clinical practices based on the theory of TCM. FGWYD is composed of Aconiti Lateralis Radix Praeparata 15g, Zingiberis Rhizoma $15 \mathrm{~g}$, Acoritataninowii Rhizoma 15g, Epimrdii Herba $15 \mathrm{~g}$, Cinnamomi Ramulus $15 \mathrm{~g}$, Morindae Officinalis Radix $15 \mathrm{~g}$, Arum Ternatum Thunb. $15 \mathrm{~g}$, Panax Ginseng C. A. Mey. $15 \mathrm{~g}$, Panax Notoginseng (Burk.) F. H. Chen Ex C. Chow $15 \mathrm{~g}$, and Radix Rhei Et Rhizome 6g. The multicenter clinical randomized controlled trial confirmed that the FGWYD group was superior to the monadipine tablet group in terms of the recovery of daily living ability and brain nerve mediators (such as norepinephrine, dopamine, and etocholine) [14, 15]. Our previous research found that FGWYD can inhibit the overexpression of CDK5 and thereby inhibit the hyperphosphorylation of Tau protein to reduce brain nerve fiber tangles, significantly improving the cognitive ability of SAMP8 mice [16]. However, previous studies have mainly focused on the study of a single signaling pathway, and there is a lack of systematic research. More importantly, due to the characteristics of TCM's "multiple compounds and multiple targets," it is difficult to explore its overall efficacy. Therefore, this study will integrate network pharmacology and proteomics 
TABLE 1: The oral absorbable and pharmacologically active components.

\begin{tabular}{|c|c|c|c|c|c|c|}
\hline Mol ID & Molecule name & MW & $\mathrm{OB}(\%)$ & Caco-2 & DL & Structure \\
\hline MOL001506 & Supraene & 410.8 & 33.54594 & 2.08183 & 0.42161 & \\
\hline MOL002883 & Ethyl oleate (NF) & 310.58 & 32.39739 & 1.40295 & 0.19061 & \\
\hline MOL009525 & 3beta-24S(R)-butyl-5-alkenyl-cholestol & 456.88 & 35.35249 & 1.36151 & 0.82221 & \\
\hline MOL009524 & 3beta,20(R),5-alkenyl-stigmastol & 414.79 & 36.91391 & 1.35994 & 0.75074 & \\
\hline MOL000358 & Beta-sitosterol & 414.79 & 36.91391 & 1.32463 & 0.75123 & \\
\hline MOL000359 & Sitosterol & 414.79 & 36.91391 & 1.32059 & 0.7512 & \\
\hline MOL002879 & Diop & 390.62 & 43.59333 & 0.7934 & 0.39247 & \\
\hline MOL006147 & Alizarin-2-methylether & 254.25 & 32.80877 & 0.61959 & 0.20971 & \\
\hline
\end{tabular}


TABle 1: Continued.

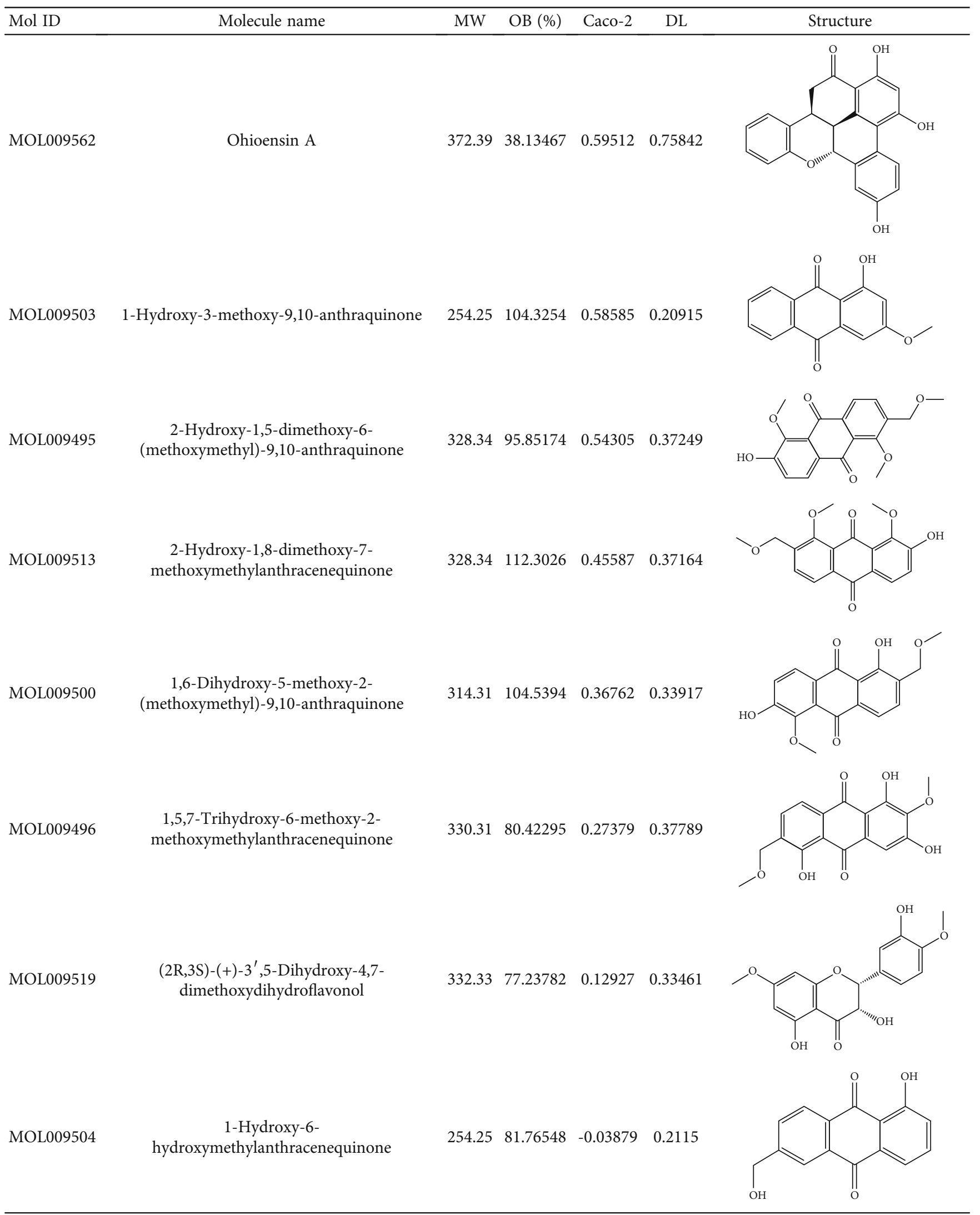


TABLE 1: Continued.

\begin{tabular}{|c|c|c|c|c|c|c|}
\hline Mol ID & Molecule name & MW & OB (\%) & Caco-2 & DL & Structure \\
\hline MOL009537 & Americanin A & 328.34 & 46.70571 & -0.07814 & 0.34901 & \\
\hline MOL009551 & Isoprincepin & 494.53 & 49.12132 & -0.18169 & 0.77375 & \\
\hline MOL001755 & 24-Ethylcholest-4-en-3-one & 412.77 & 36.08361 & 1.4563 & 0.75703 & \\
\hline MOL002670 & Cavidine & 353.45 & 35.64183 & 1.0817 & 0.80513 & \\
\hline MOL002714 & Baicalein & 270.25 & 33.51892 & 0.63086 & 0.20888 & \\
\hline MOL000449 & Stigmasterol & 412.77 & 43.82985 & 1.44458 & 0.75665 & \\
\hline MOL005030 & Gondoic acid & 310.58 & 30.70294 & 1.20473 & 0.19743 & \\
\hline MOL000519 & Coniferin & 314.41 & 31.11 & 0.42439 & 0.32308 & \\
\hline MOL006936 & 10,13-Eicosadienoic & 308.56 & 39.99355 & 1.22213 & 0.20012 & \\
\hline MOL006937 & $\begin{array}{l}\text { 12,13-Epoxy-9-hydroxynonadeca-7,10-dienoic } \\
\text { acid }\end{array}$ & 324.51 & 42.15218 & 0.17979 & 0.24248 & \\
\hline
\end{tabular}


TABle 1: Continued.

\begin{tabular}{|c|c|c|c|c|c|c|}
\hline Mol ID & Molecule name & MW & OB (\%) & Caco-2 & DL & Structure \\
\hline MOL006957 & $\begin{array}{c}(3 \mathrm{~S}, 6 \mathrm{~S})-3-(\text { Benzyl)-6-(4- } \\
\text { hydroxybenzyl)piperazine-2,5-quinone }\end{array}$ & 310.38 & 46.8889 & 0.41366 & 0.26989 & \\
\hline MOL003578 & Cycloartenol & 426.8 & 38.68566 & 1.52617 & 0.78093 & \\
\hline MOL002388 & Delphin & 303.26 & 57.7617 & 0.11969 & 0.2786 & \\
\hline MOL002415 & 6-Demethyldesoline & 453.64 & 51.87164 & -0.25991 & 0.65822 & \\
\hline MOL002419 & (R)-Norcoclaurine & 271.34 & 82.54295 & 0.62871 & 0.20872 & \\
\hline MOL002397 & Karakoline & 377.58 & 51.7309 & 0.32469 & 0.73447 & \\
\hline MOL002421 & Ignavine & 449.59 & 84.07948 & -0.07071 & 0.24798 & \\
\hline
\end{tabular}


TABle 1: Continued.

\begin{tabular}{|c|c|c|c|c|c|c|}
\hline Mol ID & Molecule name & MW & OB (\%) & Caco-2 & $\mathrm{DL}$ & Structure \\
\hline MOL002422 & Isotalatizidine & 407.61 & 50.82414 & -0.10596 & 0.73291 & \\
\hline MOL002395 & Deoxyandrographolide & 334.5 & 56.3041 & 0.181 & 0.31451 & \\
\hline MOL002410 & Benzoylnapelline & 463.67 & 34.0565 & 0.19203 & 0.52933 & \\
\hline MOL002416 & Deoxyaconitine & 629.82 & 30.95922 & -0.2338 & 0.24469 & \\
\hline MOL002434 & Carnosifloside I & 456.78 & 38.15575 & 0.2846 & 0.79654 & \\
\hline MOL000538 & Hypaconitine & 615.79 & 31.38846 & -0.3365 & 0.26085 & \\
\hline MOL002211 & 11,14-Eicosadienoic acid & 308.56 & 39.99355 & 1.21793 & 0.20044 & \\
\hline
\end{tabular}


TABle 1: Continued.

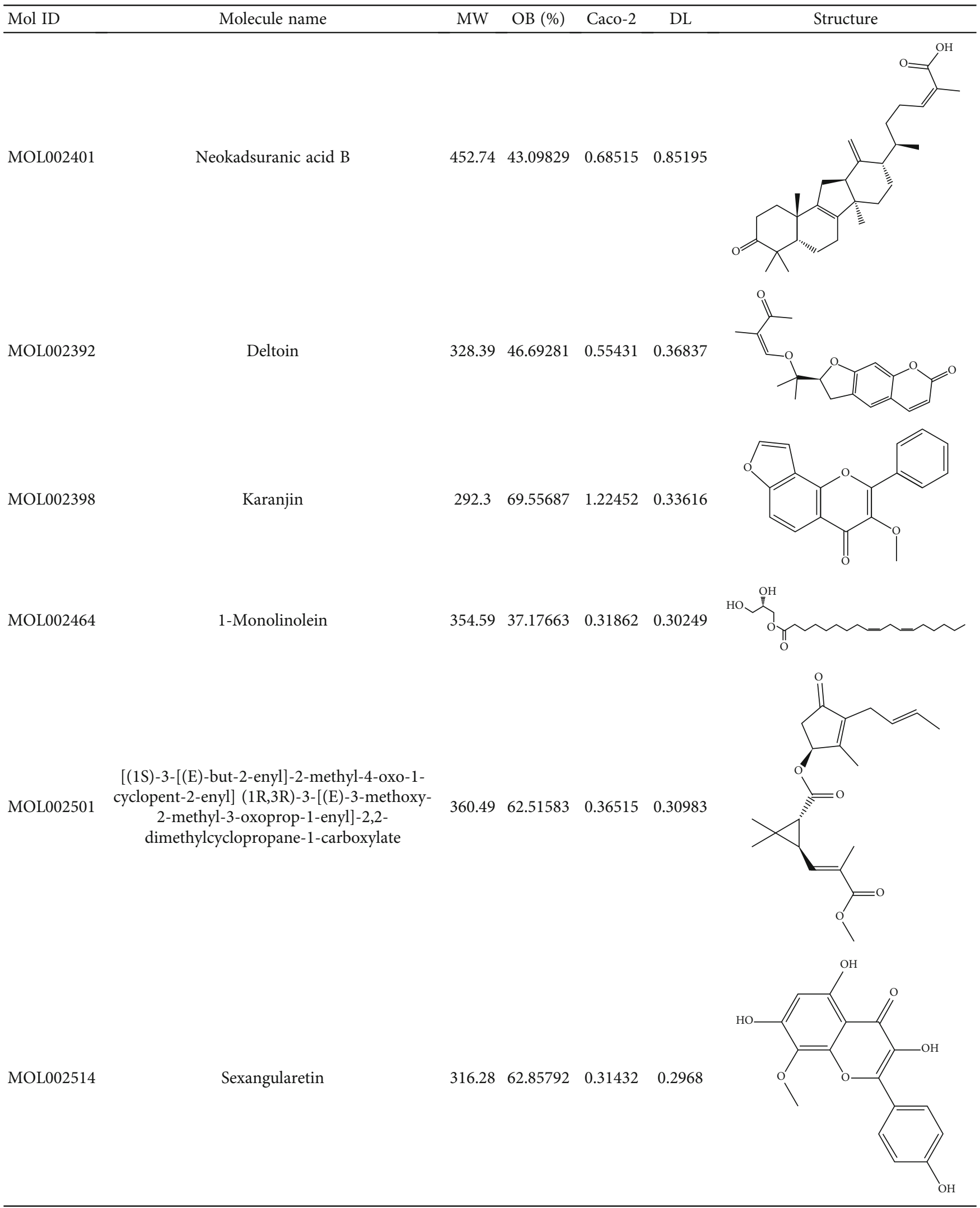


TABLE 1: Continued.

\begin{tabular}{|c|c|c|c|c|c|c|}
\hline Mol ID & Molecule name & MW & OB (\%) & Caco-2 & DL & Structure \\
\hline MOL001736 & (-)-Taxifolin & 304.27 & 60.50622 & -0.24278 & 0.27342 & \\
\hline MOL000492 & $(+)$-Catechin & 290.29 & 54.82643 & -0.03424 & 0.24164 & \\
\hline MOL000073 & ent-Epicatechin & 290.29 & 48.95984 & 0.01948 & 0.24162 & \\
\hline MOL004576 & Taxifolin & 304.27 & 57.84156 & -0.22844 & 0.27345 & \\
\hline MOL011169 & Peroxyergosterol & 428.72 & 44.39152 & 0.86327 & 0.82 & \\
\hline MOL001510 & 24-Epicampesterol & 400.76 & 37.57682 & 1.43482 & 0.71413 & \\
\hline MOL001645 & Linoleyl acetate & 308.56 & 42.10077 & 1.35826 & 0.19845 & \\
\hline MOL001771 & Poriferast-5-en-3beta-ol & 414.79 & 36.91391 & 1.45001 & 0.75034 & \\
\hline
\end{tabular}


TABLE 1: Continued.

\begin{tabular}{|c|c|c|c|c|c|c|}
\hline Mol ID & Molecule name & MW & OB (\%) & Caco-2 & $\mathrm{DL}$ & Structure \\
\hline MOL001792 & Liquiritigenin & 256.27 & 32.76272 & 0.50823 & 0.18316 & \\
\hline MOL003044 & Chryseriol & 300.28 & 35.85089 & 0.39361 & 0.27415 & \\
\hline MOL003542 & 8-Isopentenyl-kaempferol & 354.38 & 38.04434 & 0.53297 & 0.3948 & \\
\hline MOL000422 & Kaempferol & 286.25 & 41.88225 & 0.26096 & 0.24066 & \\
\hline MOL004367 & Olivil & 376.44 & 62.2286 & -0.1612 & 0.40642 & \\
\hline MOL004373 & Anhydroicaritin & 368.41 & 45.41193 & 0.72306 & 0.43786 & \\
\hline MOL004380 & $\begin{array}{c}\text { C-Homoerythrinan, 1,6-didehydro-3,15,16- } \\
\text { trimethoxy-, (3.beta.)- }\end{array}$ & 329.48 & 39.13993 & 1.01828 & 0.49461 & \\
\hline
\end{tabular}


TABle 1: Continued.

\begin{tabular}{|c|c|c|c|c|c|c|}
\hline Mol ID & Molecule name & MW & OB $(\%)$ & Caco-2 & DL & Structure \\
\hline MOL004382 & Yinyanghuo A & 420.49 & 56.95738 & 0.37565 & 0.76747 & \\
\hline MOL004384 & Yinyanghuo $\mathrm{C}$ & 336.36 & 45.672 & 0.74533 & 0.50155 & \\
\hline MOL004386 & Yinyanghuo E & 352.36 & 51.63213 & 0.50883 & 0.5474 & \\
\hline MOL004388 & $\begin{array}{c}\text { 6-Hydroxy-11,12-dimethoxy-2,2-dimethyl-1,8- } \\
\text { dioxo-2,3,4,8-tetrahydro-1H-isochromeno[3,4- } \\
\text { h]isoquinolin-2-ium }\end{array}$ & 370.41 & 60.64151 & 0.34258 & 0.65693 & \\
\hline MOL004391 & 8-(3-Methylbut-2-enyl)-2-phenyl-chromone & 290.38 & 48.5445 & 1.52596 & 0.25066 & \\
\hline MOL004396 & $\begin{array}{l}\text { 1,2-bis(4-Hydroxy-3-methoxyphenyl)propan- } \\
\text { 1,3-diol }\end{array}$ & 320.37 & 52.31425 & 0.0015 & 0.22066 & \\
\hline
\end{tabular}


TABle 1: Continued.

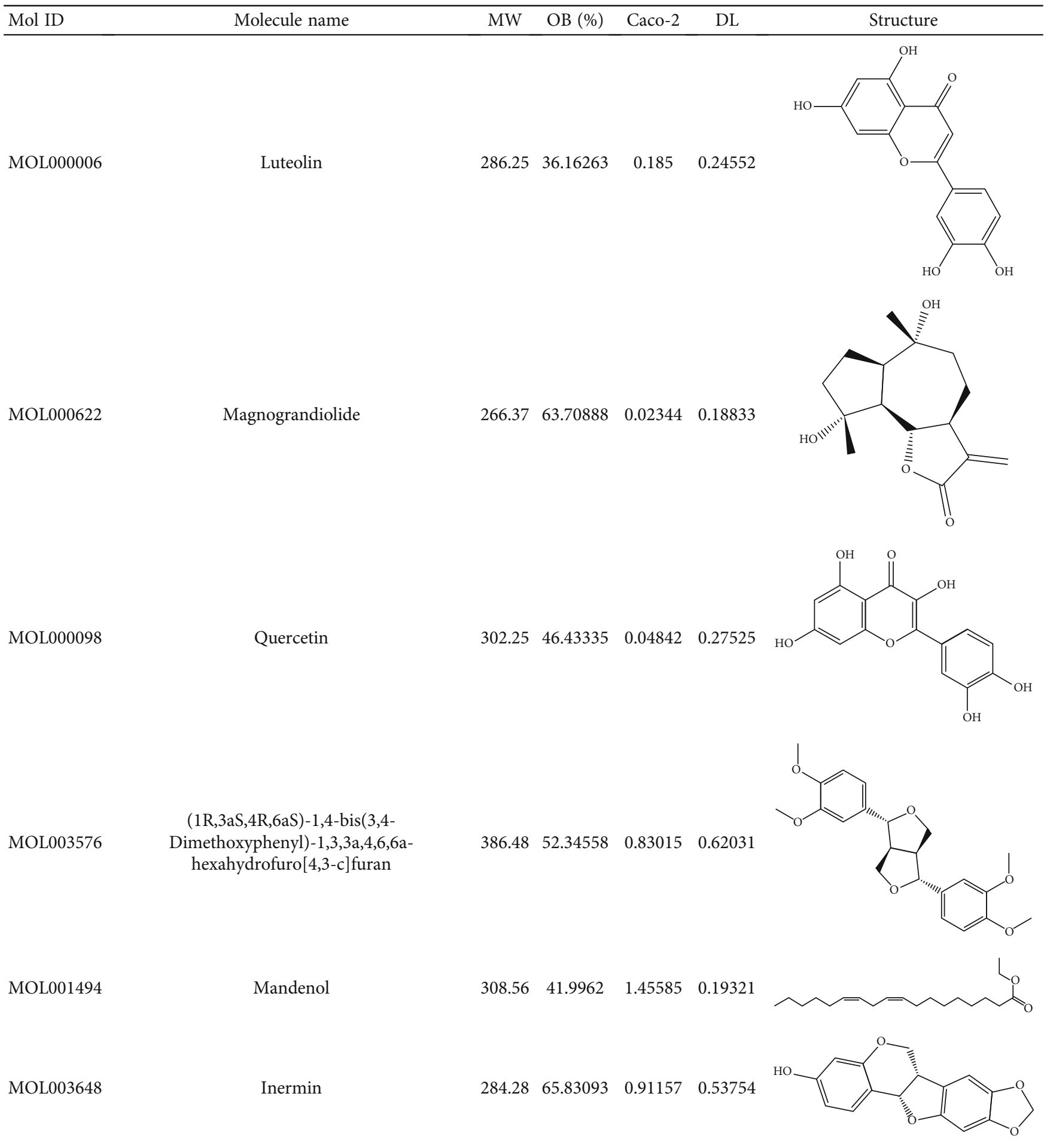


TABLE 1: Continued.

\begin{tabular}{|c|c|c|c|c|c|c|}
\hline Mol ID & Molecule name & MW & OB (\%) & Caco-2 & DL & Structure \\
\hline MOL004492 & Chrysanthemaxanthin & 584.96 & 38.72398 & 0.50972 & 0.58352 & \\
\hline MOL005308 & Aposiopolamine & 271.34 & 66.64691 & 0.65617 & 0.21999 & \\
\hline MOL005314 & Celabenzine & 379.55 & 101.8826 & 0.77185 & 0.48772 & \\
\hline MOL005317 & Deoxyharringtonine & 515.66 & 39.27444 & 0.18714 & 0.8116 & \\
\hline MOL005318 & Dianthramine & 289.26 & 40.44641 & -0.22511 & 0.19676 & \\
\hline
\end{tabular}


TABle 1: Continued.

\begin{tabular}{|c|c|c|c|c|c|c|}
\hline Mol ID & Molecule name & MW & OB (\%) & Caco-2 & $\mathrm{DL}$ & Structure \\
\hline MOL005320 & Arachidonate & 304.52 & 45.57325 & 1.26865 & 0.20491 & \\
\hline MOL005321 & Frutinone A & 264.24 & 65.90373 & 0.88838 & 0.34184 & \\
\hline MOL005348 & Ginsenoside-Rh4 & 458.8 & 31.11215 & 0.49755 & 0.77829 & \\
\hline MOL005356 & Girinimbin & 263.36 & 61.2153 & 1.72097 & 0.31484 & \\
\hline MOL005357 & Gomisin B & 514.62 & 31.99042 & 0.60183 & 0.82858 & \\
\hline MOL005360 & Malkangunin & 432.56 & 57.71384 & 0.21673 & 0.62642 & \\
\hline MOL005376 & Panaxadiol & 460.82 & 33.08796 & 0.82469 & 0.79404 & \\
\hline
\end{tabular}


TABLE 1: Continued.

\begin{tabular}{|c|c|c|c|c|c|c|}
\hline Mol ID & Molecule name & MW & OB (\%) & Caco-2 & $\mathrm{DL}$ & Structure \\
\hline MOL005384 & Suchilactone & 368.41 & 57.51882 & 0.82023 & 0.55573 & \\
\hline MOL005399 & Alexandrin & 414.79 & 36.91391 & 1.30404 & 0.75268 & \\
\hline MOL005401 & Ginsenoside Rg5 & 442.8 & 39.56307 & 0.87742 & 0.78506 & \\
\hline MOL000787 & Fumarine & 353.4 & 59.2625 & 0.56266 & 0.82694 & \\
\hline
\end{tabular}

strategies to comprehensively analyze the mechanism of FGWYD regulating VD biological network. The idea of this research is shown in Figure 1.

\section{Materials and Methods}

2.1. FGWYD Potential Component Collection. TCMSP (https://tcmspw.com/tcmsp.php) [17] was used to search for keywords such as "Aconiti Lateralis Radix Praeparata" and "Zingiberis Rhizoma" to collect the chemical components in FGWYD and the pharmacokinetic parameters of each chemical component. Oral bioavailability $(\mathrm{OB}) \geq 30 \%$, Caco- $2>-$ 0.4 , and drug-likeness $(\mathrm{DL}) \geq 0.18$ were used as the screening thresholds to screen the oral absorbable and pharmacologically active components in FGWYD [17] (Table 1).

2.2. FGWYD Targets and VD Genes. The targets of the FGWYD components were collected from TCMSP [18]. The VD genes were collected from the OMIM database (http://omim.org/) [19], Genecards (http://www.genecards .org) [20], and references [21-25]. The UniProt database (https://www.uniprot.org/) is used to convert target protein names into corresponding official gene names (Table S1 and Table S2).

2.3. Network Construction and Analysis Methods. FGWYD potential targets and VD genes were imported into the String database (https://string-db.org/) [26], the species was limited to "Homo sapiens," isolated targets were removed, and the protein-protein interaction (PPI) data was obtained based on the confidence level $\geq 0.4$. The analysis results were saved as TSV format files and imported into Cytoscape 3.7.0 software for network construction. The tightly connected part of the PPI network is considered a cluster. In a biological network, cluster may represent a biological module related to disease occurrence or drug treatment. The cluster in the PPI network was detected by MCODE [27]. The target protein gene list was imported into the DAVID database (https:// david.ncifcrf.gov/summary.jsp) [28], the species was limited to "Homo sapiens," and Gene Ontology (GO) enrichment analysis and KEGG (Kyoto Encyclopedia of Genes and Genomes) signaling pathway analysis were performed. 
TABLE 2: The oligonucleotide sequences.

\begin{tabular}{lccc}
\hline Gene & Method & Forward sequence & Reverse sequence \\
\hline Nrf2/ARE oligonucleotide & EMSA & TTTATGCTGTGTCATGGTT & AACCATACACAGCATAAAA \\
Nrf2/mutARE oligonucleotide & EMSA & TTTTATGCAGACACATGGTT & AACCATACTGTCTATAAAA \\
\hline
\end{tabular}

TABLE 3: The primer.

\begin{tabular}{lc}
\hline Gene & \multicolumn{1}{c}{ Sequence } \\
\hline \multirow{2}{*}{ HO-1 } & F: AGAGGGTGATAGAAGAGGCCAA \\
& P: GTGTAAGGACCCATCGGAGAAG \\
$\beta$-Actin & F: AGGGGCCGOACTCGTCATACT \\
& P: GGCGGCACCACCATGTACCCT \\
\hline
\end{tabular}

\subsection{Experimental Materials}

2.4.1. Experimental Animal. Sixty (60) male SD rats that were specific pathogen-free (SPF) grade were purchased and placed in the Experimental Animal Center of Hunan University of Chinese Medicine (Qualification Certificate No.: HNASLKJ20113616). Animal experiments were approved by the Animal Ethics Committee of Hunan University of Chinese Medicine and were in accordance with the National Institute of Health's Guide for the Care and Use of Laboratory Animals.

2.4.2. Experimental Drugs. FGWYD is composed of Aconiti Lateralis Radix Praeparata 15g, Zingiberis Rhizoma 15g, Acoritataninowii Rhizoma 15 g, Epimrdii Herba 15 g, Cinnamomi Ramulus $15 \mathrm{~g}$, Morindae Officinalis Radix $15 \mathrm{~g}$, Arum Ternatum Thunb. 15 g, Panax Ginseng C. A. Mey. 15 g, Panax Notoginseng (Burk.) F. H. Chen Ex C. Chow $15 \mathrm{~g}$, and Radix Rhei Et Rhizome $6 \mathrm{~g}$. The medicinal materials were purchased from the Pharmaceutical Factory of the First Affiliated Hospital of Hunan University of Chinese Medicine. The medicinal materials of FGWYD were extracted twice; the first time was extracted with 10 times the volume of water for 2 hours, and the second time was extracted with 8 times the volume of water for 1.5 hours. The medicinal solution is filtered, combined, and concentrated to contain crude drug $1 \mathrm{~g} / \mathrm{mL}$, stored at $4^{\circ} \mathrm{C}$. During the experiment, FGWYD was diluted with double-distilled water to an appropriate concentration. Piracetam tablets (Naofukang, NFK) were purchased from Shanghai Xinyi Pharmaceutical Co., Ltd. (Guo Yao Zhunzi H31020714). The reference substance benzoylaconitine (batch number: CHB190207), benzoylmesaconine (batch number: CHB200120), and benzoylhypacoitine (batch number: CHB200201) were purchased from Chengdu Croma Biotechnology Co., Ltd. The quality scores of all controls were $\geq 98 \%$.

2.4.3. Reagents and Instruments. Instruments: electrothermal constant temperature incubator (Shanghai Yuejin Medical Devices Co., Ltd., HH.B11.360), electrothermal constant drying oven (Shanghai Yuejin Medical Devices Co., Ltd., GZXDH.400), UVP gel imaging system (Thermo Fisher Scientific, CA91786 USA UVP GDS-8000 System), electrophoresis system (Beijing Liuyi Biological Technology Co., Ltd., DYCZ24DN), semidry film transfer system (ATTO, WSE-4040),
TABLE 4: Sample marking information.

\begin{tabular}{lc}
\hline Sample & Label \\
\hline CK1 & 113 \\
CK2 & 114 \\
HD1 & 115 \\
HD2 & 116 \\
M1 & 117 \\
M2 & 118 \\
W1 & 119 \\
W2 & 121 \\
\hline
\end{tabular}

transfer decolorization shaker (Haimen Qilin Bell Instrument Manufacturing Co., Ltd., TS-8), fluorescence quantitative PCR instrument (ABI, 7500), vortex oscillator (Haimen Qilin Bell Instrument Company, QL-902), and horizontal electrophoresis instrument (Beijing Liuyi Biotechnology Co., Ltd.).

Reagents: RIPA lysate (Beijing Soleil, 80010), SuperReal PreMix Plus (SYBR Green) (Tiangen Biotechnology Co., Ltd.), and DL2000 DNA Marker (TAKARA, 3427A). The malondialdehyde (MDA) determination kit, the Total Antioxidant Capacity (T-AOC) Test Kit, the Lipid Peroxide (LPO) Test Kit, the Glutathione Peroxidase (GSH-Px) Test Kit, and the superoxide dismutase (SOD) determination kit were purchased from Nanjing Jiancheng Bioengineering Company. High-fat feed formula was composed of the following: $3 \%$ cholesterol, $0.5 \%$ sodium cholate, $0.2 \%$ propylthiouracil, $5 \%$ sugar, $10 \%$ lard, and $81.3 \%$ basic feed.

\subsection{Experimental Methods}

\subsubsection{FGWYD Quality Control}

(1) Preparation of FGWYD solution: FGWYD $25 \mathrm{~mL}$ is precisely pipetted into a round-bottom flask and evaporated to dryness. The residue of FGWYD was accurately combined with $20 \mathrm{~mL}$ chloroform, $4 \mathrm{~mL}$ ammonia, and $4 \mathrm{~mL}$ methanol; heated to reflux for $2 \mathrm{~h}$; cooled and filtered; then washed with chloroform 3 times, combined with the FGWYD filtrate, and evaporated to dryness in a $60^{\circ} \mathrm{C}$ water bath

(2) Preparation of reference solution: benzoylmesaconine, benzoylaconitine, and benzoylhypacoitine $2.81,2.33$, and $2.75 \mathrm{mg}$ were accurately weighed, respectively, and $0.05 \%$ hydrochloric acid-methanol was added to prepare $0.562,0.466$, and $0.550 \mathrm{~g} / \mathrm{L}$ benzoylmesaconine, benzoylaconitine, and benzoylhypacoitine reference solutions

(3) HPLC condition: Waters XTerra MS C18 column $(150 \mathrm{~mm} \times 4.6 \mathrm{~mm}, 5 \mu \mathrm{m})$, XTerra MS C18 guard 


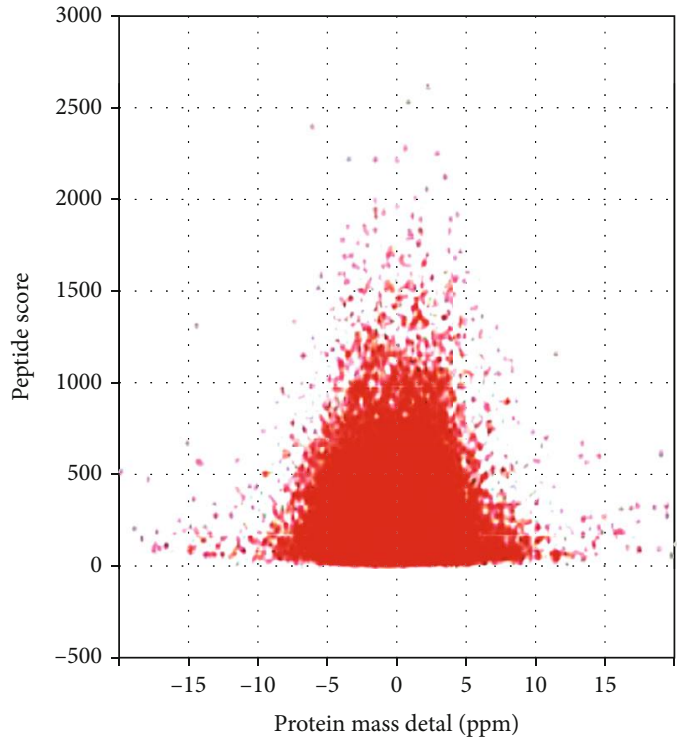

(a)

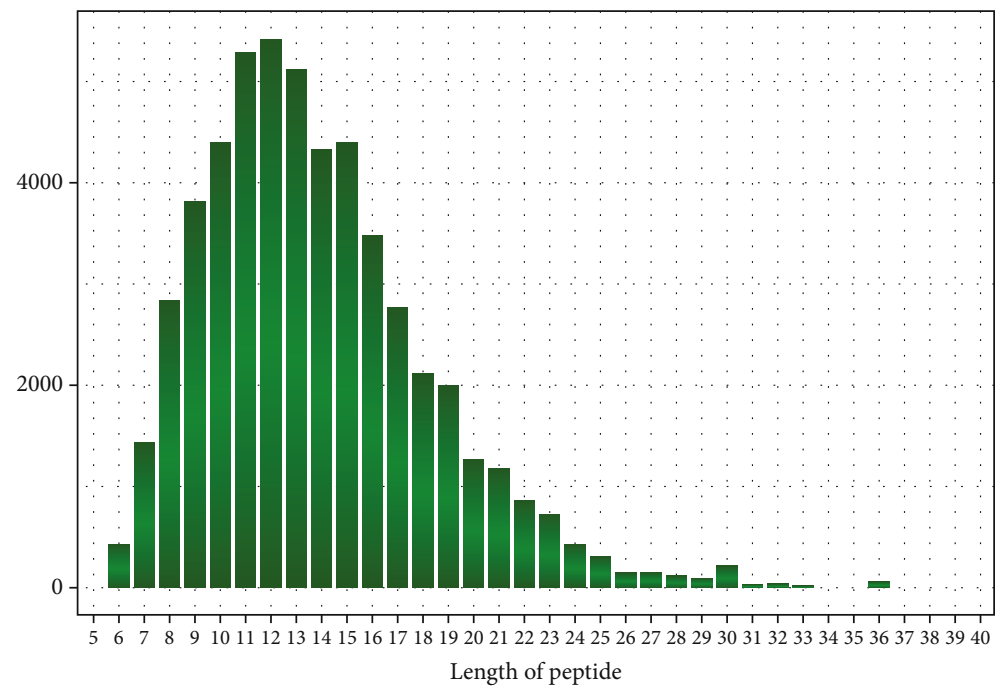

(b)

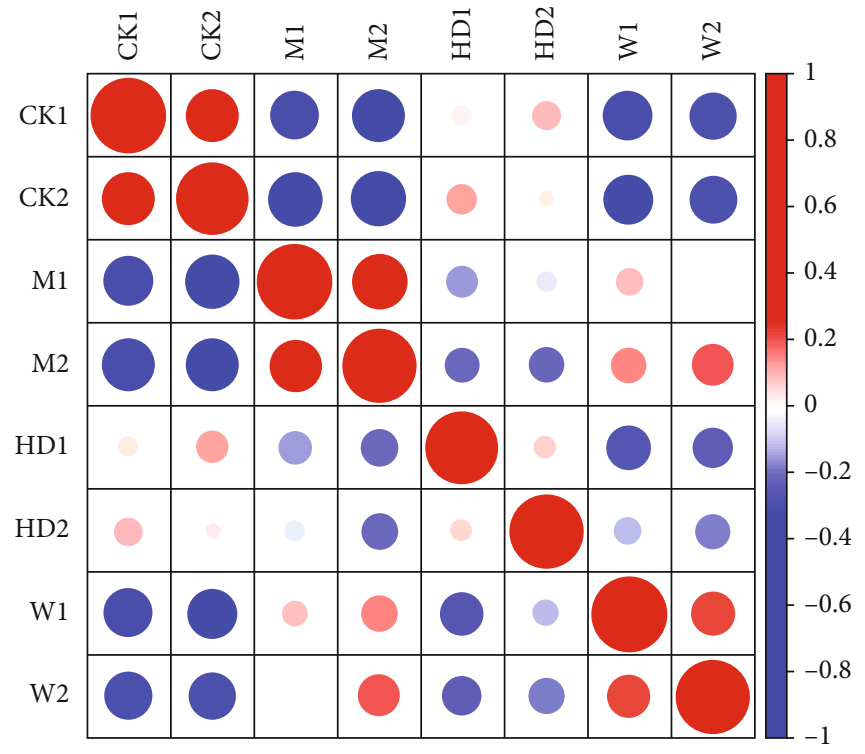

(c)

Figure 2: Mass spectrometry quality control ((a) quality bias distribution of peptide segment; (b) identified peptide distribution; (c) sample repeatability test; CK1: control group 1; CK2: control group 2; M1: model group 1; M2: model group 2; HD1: FGWYD group 1; HD2: FGWYD group 2; W1: positive control group 1; W2: positive control group 2).

column $(20 \mathrm{~mm} \times 4.6 \mathrm{~mm}, 5 \mu \mathrm{m})$. Mobile phase: acetonitrile (a) $-0.1 \mathrm{~mol} \cdot \mathrm{L}^{-1}$ ammonium acetate solution $(\mathrm{pH}=6.97)(\mathrm{b})$; gradient elution; flow rate: $1 \mathrm{~mL} \cdot \mathrm{min}^{-}$ 1 . Detection wavelength: $235 \mathrm{~nm}$. Injection volume: $10 \mu \mathrm{L}$; detection temperature: room temperature

The HPLC results showed that the content of the components in FGWYD was benzoylmesaconine $0.14928 \mathrm{mg} / \mathrm{g}$, benzoylaconitine $0.014736 \mathrm{mg} / \mathrm{g}$, and benzoylhypacoitine $0.021648 \mathrm{mg} / \mathrm{g}$ (Figure S1).

2.5.2. Animal Modeling. The rats were fed adaptively for 5 days. On the sixth day, a water maze test was performed to exclude rats with swimming disorders and rats that failed to find a platform. A total of 3 rats were removed. Then, the rats were randomly grouped: 10 rats in the sham operation group, and the remaining 50 rats entered the modeling group. The experimental group was fed a high-fat diet, and at the same time, a one-time intraperitoneal injection of vitamin D3 700,000 IU/kg at the beginning of feeding. The sham operation group was fed basic feed and given the same volume of saline. After the success of the atherosclerosis (AS) model, on the basis of the AS model, the rat VD model was prepared by the bilateral common carotid artery clipping and reperfusion method. In rats from the sham operation group, only neck skin incision was performed without ischemic surgery. The cages were marked with numbers as groups, and intervention drugs were also marked with 
numbers. The meaning of the number will be kept by a third person before the end of the experiment. Animal experiment operators, data collectors, and statistical analysts were not aware of grouping and intervention drugs.

2.5.3. Animal Grouping and Intervention. One week after modeling, the rats were given intragastric administration after the surgical incision of the rats was completely healed. Fifty (50) successful rats were randomly divided into 5 groups: the FGWYD low-dose group (FGWYD-L, 10 rats), the FGWYD medium-dose group (FGWYD-M, 10 rats), the FGWYD high-dose group (FGWYD-H, 10 rats), the positive control group (NFK, 10 rats), and the model group (10 rats).

The intervention began 4 weeks after modeling, and the dosage was calculated based on the ratio of human and rat body surface area coefficient. The FGWYD low-dose group was given $1.25 \mathrm{~g}$ of crude drug $/ \mathrm{kg}$, the FGWYD mediumdose group was given $2.5 \mathrm{~g}$ of crude $\mathrm{drug} / \mathrm{kg}$, and the FGWYD high-dose group was given $5 \mathrm{~g}$ of crude $\mathrm{drug} / \mathrm{kg}$. The positive drug control group was given piracetam $0.15 \mathrm{~g} / \mathrm{kg}$. The sham operation group and the model group were given intragastric administration with the corresponding volume of double-distilled water. The administration lasted 14 days. Two rats died in the model group, FGWYD high-dose group, and positive control group, respectively. Morris water maze experiment was used to test learning and memory ability. HE staining was used to observe the pathological changes of brain tissue.

2.5.4. Morris Water Maze Behavior Test. The pool was equally divided into four quadrants and marked, and four quadrant entry points are marked on the inner wall of the pool, and a platform is placed at the center of the third quadrant of the pool. The pool was filled with water up to about $2 \mathrm{~cm}$ above the platform. The ink is added to the water and mixed well to hide the platform. The water in the pool is heated and maintained at around $22^{\circ} \mathrm{C}$. A small fixed camera is installed above the pool to track and record the swimming trajectory of rats. The content of Morris water maze detection includes a positioning navigation experiment and a space exploration experiment.

2.5.5. Detection of Nrf2 and HO-1 Protein Expression by Western Blot. After the water maze experiment on rats was completed, fresh brain was collected under low temperature under anesthesia with $1 \%$ sodium pentobarbital $35 \mathrm{mg} / \mathrm{kg}$. After extracting the total protein and measuring the concentration, SDS-PAGE electrophoresis was performed. After the transfer, the color was developed with ECL color-developing solution.

2.5.6. NRF2 and ARE Binding Force Test by Electrophoretic Mobility Shift Assay (EMSA). EMSA was performed after extracting the nucleoprotein. First, the oligonucleotide probe is denatured and annealed into a double strand $\left(94^{\circ} \mathrm{C}\right.$ for $5 \mathrm{~min}$, gradually returning to room temperature), and the annealing effect is checked by $12 \%$ PAGE gel electrophoresis, diluted and aliquoted, and stored at $-20^{\circ} \mathrm{C}$ for use. The EMSA operation was carried out according to the operating procedures of the LightShift ${ }^{\mathrm{TM}}$ Chemiluminescent EMSA Kit

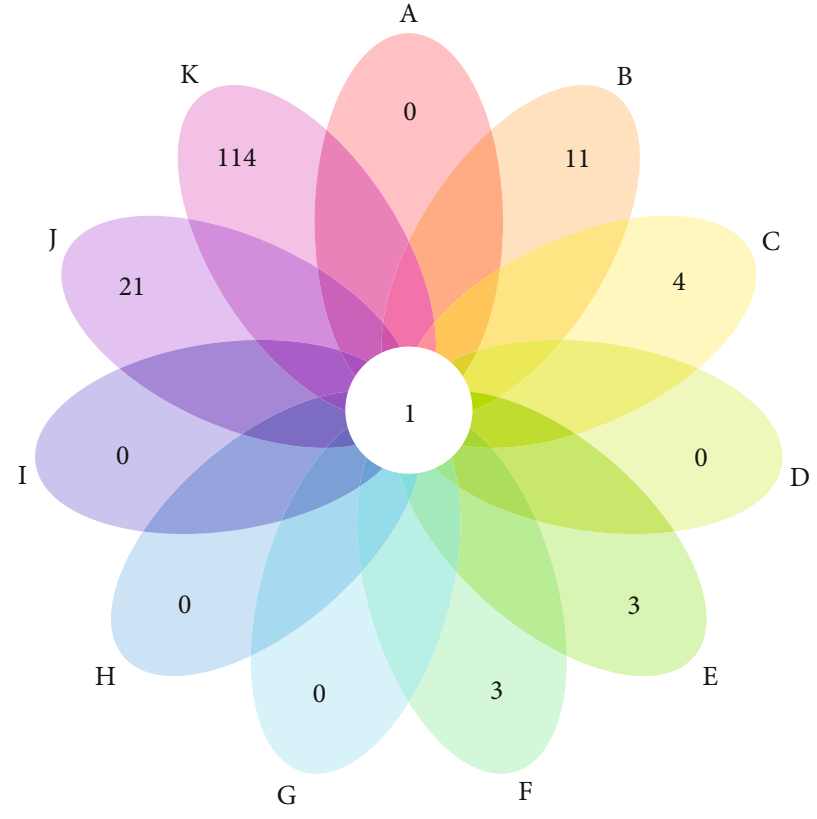

FIgURe 3: Venn diagram of FGWYD targets and VD genes ((A) Morindae Officinalis Radix; (B) Arum Ternatum Thunb.; (C) Radix Rhei Et Rhizome; (D) Aconiti Lateralis Radix Praeparata; (E) Cinnamomi Ramulus; (F) Panax Ginseng C. A. Mey.; (G) Panax Notoginseng (Burk.) F. H. Chen Ex C. Chow; (H) Zingiber Officinale Roscoe; (I) Acoritataninowii Rhizoma; (J) Epimrdii Herba; (K) VD).

(Pierce Inc.). The oligonucleotide sequences used in the experiment are shown in Table 2.

2.5.7. Detection of HO- 1 mRNA Expression by Real-Time $P C R$. Total RNA in tissue was extracted with TRIzol and was reversed transcribed into cDNA. The real-time PCR reaction system is composed of the following: $2 \mathrm{x}$ SuperReal PreMix Plus $10 \mu \mathrm{L}$, upstream primer $(10 \mu \mathrm{M}) 0.6 \mu \mathrm{L}$, downstream primer $(10 \mu \mathrm{M}) 0.6 \mu \mathrm{L}$, cDNA $100 \mathrm{mg}, 50 x$ ROX Reference Dye $0.4 \mu \mathrm{L}$, and $\mathrm{RNase}-F r e e ~ \mathrm{ddH}_{2} \mathrm{O}$ to $20 \mu \mathrm{L}$. Program setting is as follows: predenaturation at $95^{\circ} \mathrm{C}$ for $15 \mathrm{~min}$ once, at $95^{\circ} \mathrm{C}$ for $10 \mathrm{~s}$, at $58^{\circ} \mathrm{C}$ for $20 \mathrm{~s}$, and at $72^{\circ} \mathrm{C}$ for $30 \mathrm{~s}$, for 40 cycles. The primer is shown in Table 3.

2.5.8. Detection of the MDA, SOD, GSH-Px, and LPO Contents and Total Antioxidant Capacity (T-AOC) of Hippocampus. The MDA, SOD, GSH-Px, and LPO contents and $\mathrm{T}-\mathrm{AOC}$ of the hippocampus were determined strictly in accordance with the kit instructions.

\subsection{Proteomics Methods}

2.6.1. Total Protein Extraction. Under the anesthesia with $1 \%$ sodium pentobarbital $35 \mathrm{mg} / \mathrm{kg}$, the hippocampus of the rats was taken out and frozen in the refrigerator at $-80^{\circ} \mathrm{C}$. During the experiment, the rat hippocampus sample was taken out from the refrigerator at $-80^{\circ} \mathrm{C}$, put into a liquid nitrogen precooled mortar, and added liquid nitrogen to grind to a powder. Four times the volume of lysis buffer was added to the sample and placed in an ultrasonic disruptor for ultrasonic lysis. The above sample was centrifuged at 


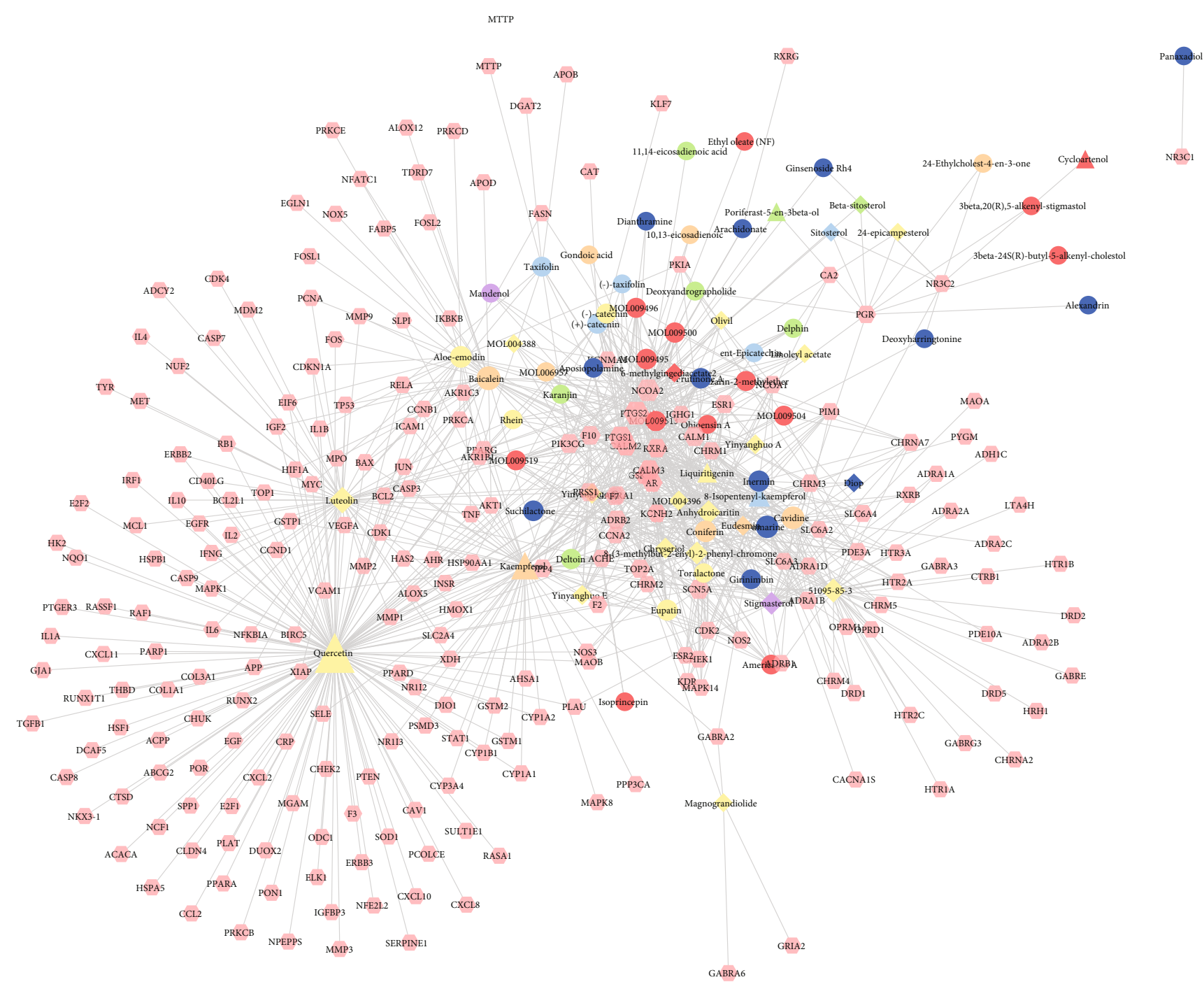

FIGURE 4: Component-target network of FGWYD. (Pink hexagons stand for FGWYD targets. Red, orange, yellow, green, blue, indigo, and purple circles stand for components of Morindae Officinalis Radix, Arum Ternatum Thunb., Radix Rhei Et Rhizome, Aconiti Lateralis Radix Praeparata, Cinnamomi Ramulus, Panax Ginseng C. A. Mey., and Panax Notoginseng (Burk.) F. H. Chen Ex C. Chow, respectively. Red, orange, and yellow diamonds stand for compounds of Zingiber Officinale Roscoe, Acoritataninowii Rhizoma, and Epimrdii Herba, respectively. Yellow diamond stands for common component of Morindae Officinalis Radix, Arum Ternatum Thunb., Radix Rhei Et Rhizome, Aconiti Lateralis Radix Praeparata, Cinnamomi Ramulus, Panax Ginseng C. A. Mey., Panax Notoginseng (Burk.) F. H. Chen Ex C. Chow, and Zingiber Officinale Roscoe. Green diamond stands for common component of Morindae Officinalis Radix, Aconiti Lateralis Radix Praeparata, Cinnamomi Ramulus, and Epimrdii Herba. Blue diamond stands for common component of Morindae Officinalis Radix, Panax Ginseng C. A. Mey., and Panax Notoginseng (Burk.) F. H. Chen Ex C. Chow. Indigo diamond stands for common component of Morindae Officinalis Radix, Panax Ginseng C. A. Mey., and Panax Notoginseng (Burk.) F. H. Chen Ex C. Chow. Purple diamond stands for common component of Arum Ternatum Thunb., Panax Ginseng C. A. Mey., Panax Notoginseng (Burk.) F. H. Chen Ex C. Chow, and Zingiber Officinale Roscoe. Red triangle stands for common component of Arum Ternatum Thunb. and Acoritataninowii Rhizoma. Orange triangle stands for common component of Panax Ginseng C. A. Mey., Acoritataninowii Rhizoma, and Epimrdii Herba. Yellow triangle stands for common component of Panax Notoginseng (Burk.) F. H. Chen Ex C. Chow and Epimrdii Herba. Green triangle stands for common component of Zingiber Officinale Roscoe and Epimrdii Herba. Blue triangle stands for common component of Acoritataninowii Rhizoma and Epimrdii Herba.)

$12000 \mathrm{~g}$ for $10 \mathrm{~min}$ at $4^{\circ} \mathrm{C}$, and the supernatant was extracted, and then the protein concentration was determined using the Bradford kit.

2.6.2. Isobaric Tags for Relative and Absolute Quantification. After the protein was digested by trypsin, the peptide was desalted with StrataXC18 (Phenomenex) and then freeze- dried in vacuo. The peptide was dissolved with 0.5 M TEAB, and then they were labeled according to the instructions of the TMT kit. The sample marking information is shown in Table 4.

2.6.3. HPLC Classification. The peptides were fractionated by high $\mathrm{pH}$ reverse HPLC, and the column was Agilent 


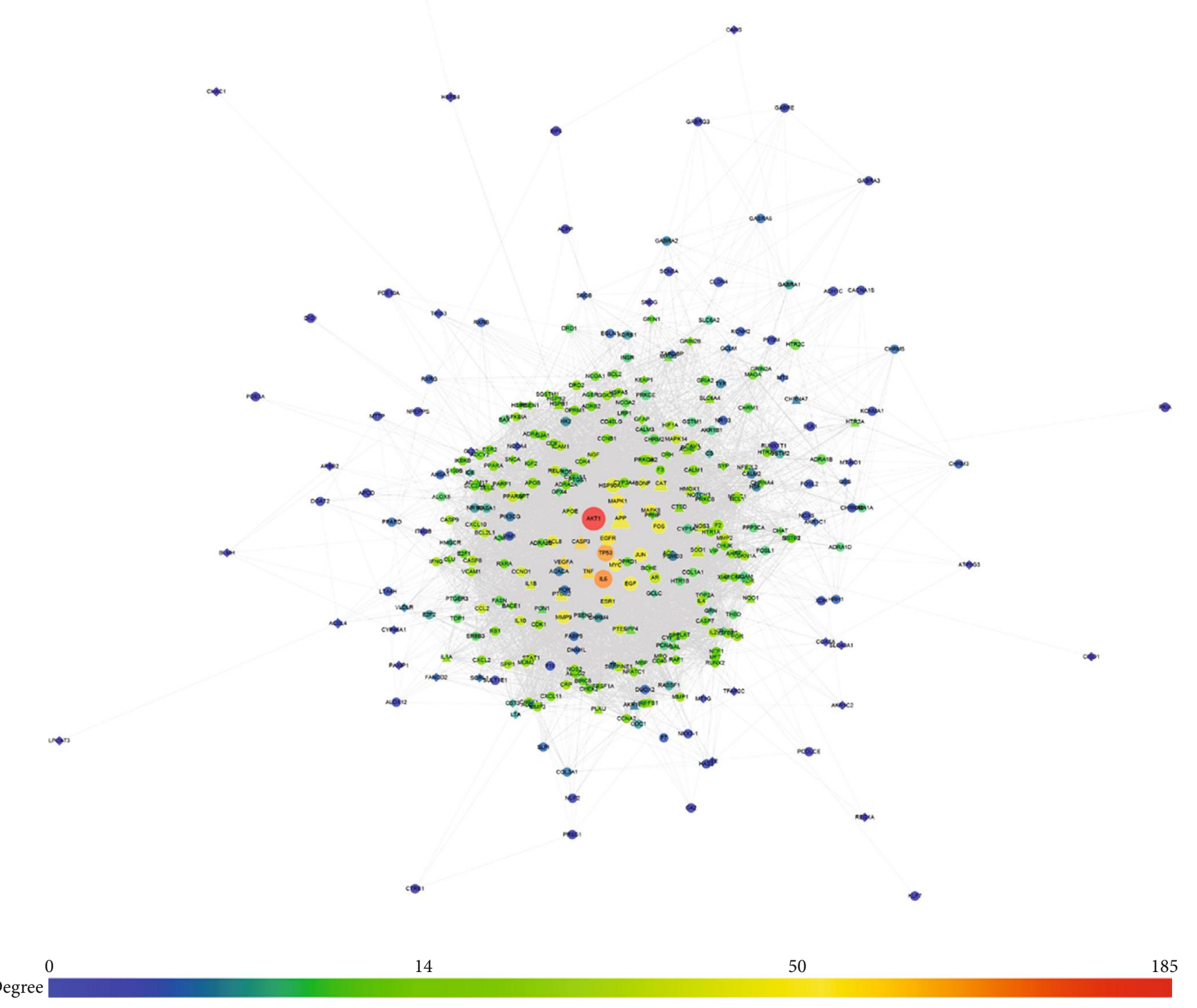

FIgURE 5: FGWYD-VD PPI network (circles stand for FGWYD targets; diamonds stand for VD genes; triangles stand for FGWYD-VD targets; the color of the nodes was related to the degree; the size of the nodes was positively related to their betweenness).

300Extend C18 (5 $\mu \mathrm{m}$ particle size, $4.6 \mathrm{~mm}$ inner diameter, $250 \mathrm{~mm}$ long).

2.6.4. Liquid Chromatography-Mass Spectrometry Analysis. The peptides were dissolved in liquid chromatography mobile phase A and separated using the EASY-nLC 1000 ultra-high-performance liquid system. After separation by ultra-high-performance liquid phase system, the peptides were injected into the NSI ion source for ionization and then analyzed by Q Exactive mass spectrometry. The secondary mass spectrometry data was retrieved using Sequest software integrated with Proteome Discoverer (version 1.3, Thermo Fisher Scientific). Retrieval parameter settings are as follows: the database is the rat proteome database in Uniprot, named
10116-PrRattusNorvegicus-0171215-9795 (Proteome ID: UP000002494) (29795 sequences).

2.6.5. Mass Spectrometry Quality Control Detection. The quality control results of mass spectrometry data are shown in Figure 2. First, we detected all the identified mass errors that were too short (mass error) (Figure 2(a)). The mass error is centered on 0 and concentrated in the range below $10 \mathrm{ppm}$, indicating that the mass error meets the requirements. Secondly, most of the peptides are distributed between 8 and 20 amino acid residues (Figure 2(b)), which is in accordance with the rule of trypsin digestion of peptides, indicating that the sample preparation meets the standard. The color in Figure 2(c) represents the correlation 


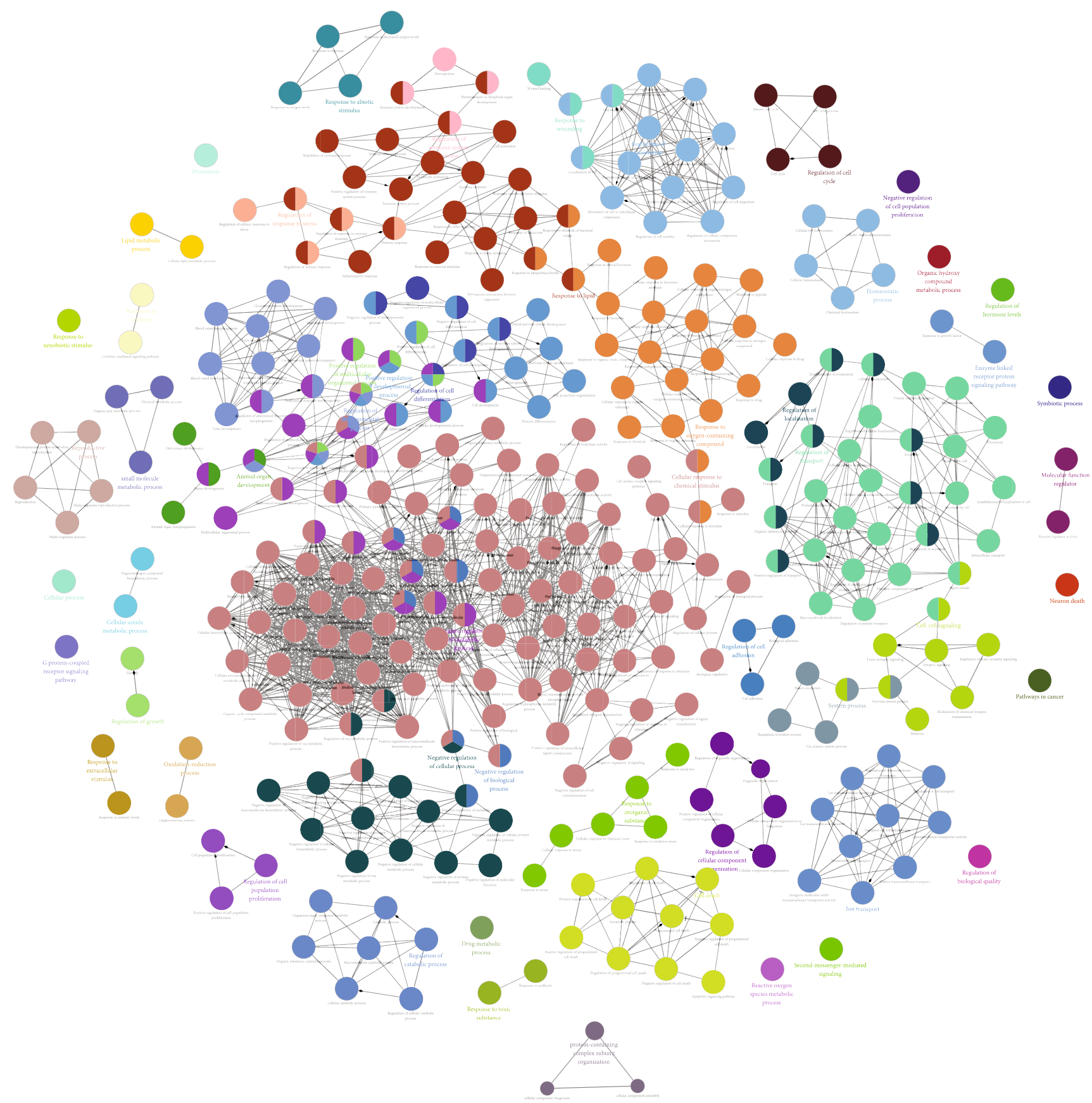

FIGURE 6: The primary enrichment analysis results visualized by ClueGO.

coefficient of protein quantification between samples. The higher the correlation coefficient between samples in the same group, the better the repeatability and the redder the color. CK1 (control group 1) and CK2 (control group 2), M1 (model group 1) and M2 (model group 2), HD1 (FGWYD group 1) and HD2 (FGWYD group 2), and W1 (positive control group 1) and W2 (positive control group 2) have higher repeatability among samples.

2.7. Statistical Analysis. All data were analyzed using SPSS17.0. Quantitative data is expressed as mean and standard deviation $(x \pm s)$. If the data conforms to the normal distribution, $t$-test analysis is used for comparison between two groups, and analysis of variance is used for comparison between multiple groups. A nonparametric test is used for data that does not conform to the normal distribution. The $\chi^{2}$ test is used for counting data, and the rank sum test is used for ranking data. $P<0.05$ was considered statistically significant.

\section{Results and Discussion}

3.1. FGWYD Targets and VD Genes. A total of 73 FGWYD components with 245 FGWYD targets were obtained from 
TABLE 5: Clusters of FGWYD-VD PPI network.

\begin{tabular}{|c|c|c|c|c|}
\hline Cluster & Score & Nodes & Edges & Targets and genes \\
\hline 1 & 44.214 & 57 & 1238 & $\begin{array}{l}\text { PLAU, MPO, CCL2, CXCL8, TGFB1, NOS2, MAPK14, SERPINE1, CAT, CASP3, BDNF, CRP, NGF, } \\
\text { CDKN1A, CXCL10, SPP1, TP53, MYC, IL1B, CCNB1, HMOX1, RELA, AKT1, FOS, MMP9, HIF1A, } \\
\text { TNFRSF1A, CD40, JUN, MAPK8, MMP1, STAT1, ICAM1, VCAM1, PTGS2, EGFR, BCL2L1, CASP9, } \\
\text { MMP2, MAPK1, IL10, PPARG, IL6, MDM2, MCL1, IL2, AR, IFNG, IL4, CD40LG, APOE, ACE, TNF, } \\
\text { MMP3, EGF, CASP8, VEGFA }\end{array}$ \\
\hline 2 & 13.778 & 46 & 310 & $\begin{array}{l}\text { NOS3, HSPA5, IKBKB, SP1, CAV1, F3, GJA1, SELE, IL1A, AGER, GSK3B, CDK2, CHEK1, PTEN, NCF1, } \\
\text { CCNA2, PARP1, KDR, CXCL2, CHEK2, HSPB1, HSPB2, HSPB3, CCND1, ESR1, HSP90AA1, RUNX2, } \\
\text { RB1, CDK1, CDK4, NFKBIA, IGFBP3, IRF1, PGR, ERBB2, CASP7, BIRC5, ADAM17, XIAP, APP, AHR, } \\
\text { IGF2, MET, NR3C1, NFE2L2, RAF1 }\end{array}$ \\
\hline 3 & 12.533 & 16 & 94 & $\begin{array}{l}\text { DPP4, DRD2, OPRM1, PTGER3, HTR1B, ADRA2A, HTR1A, APOB, ADCY2, CHRM4, CXCL11, } \\
\text { OPRD1, GAL, ADRA2C, CHRM2, ADRA2B }\end{array}$ \\
\hline 4 & 7.6 & 21 & 76 & $\begin{array}{c}\text { ADRA1B, NCOA2, PRKCA, RXRA, ADRA1D, MAOB, NCOA1, CHRM3, CYP1A1, CHRM1, HTR2A, } \\
\text { MAOA, CHRM5, GRIN1, HTR3A, PPP3CA, VIP, HRH1, ADRA1A, SNCA, HTR2C }\end{array}$ \\
\hline 5 & 5.429 & 8 & 19 & GCLM, GCLC, GSTP1, GSS, PON1, GSTM1, GSTM2, AKR1B1 \\
\hline 6 & 4.571 & 8 & 16 & GRIA2, SNCB, GABRA6, GABRG3, GABRE, CLDN4, MT3, GABRA3 \\
\hline 7 & 4.5 & 9 & 18 & SLC6A3, SYP, SLC6A4, CALM1, GRIN2A, CHRNA4, SLC6A2, CRH, CHAT \\
\hline 8 & 4.286 & 8 & 15 & ADRB1, ADRB2, PRKCD, GRIN2B, ACHE, PRKCB, DRD1, DRD5 \\
\hline 9 & 3.714 & 8 & 13 & SQSTM1, MAPT, CTSD, BACE1, PSEN1, BCL2, TFRC, CHUK \\
\hline 10 & 3.5 & 5 & 7 & AKR1C3, CYP1B1, SULT1E1, AKR1C1, NR1I2 \\
\hline 11 & 3 & 3 & 3 & F2, PLAT, THBD \\
\hline 12 & 3 & 3 & 3 & COX5A, F7, F10 \\
\hline 13 & 2.8 & 6 & 7 & GFAP, KEAP1, MBP, S100B, NQO1, SOD1 \\
\hline
\end{tabular}
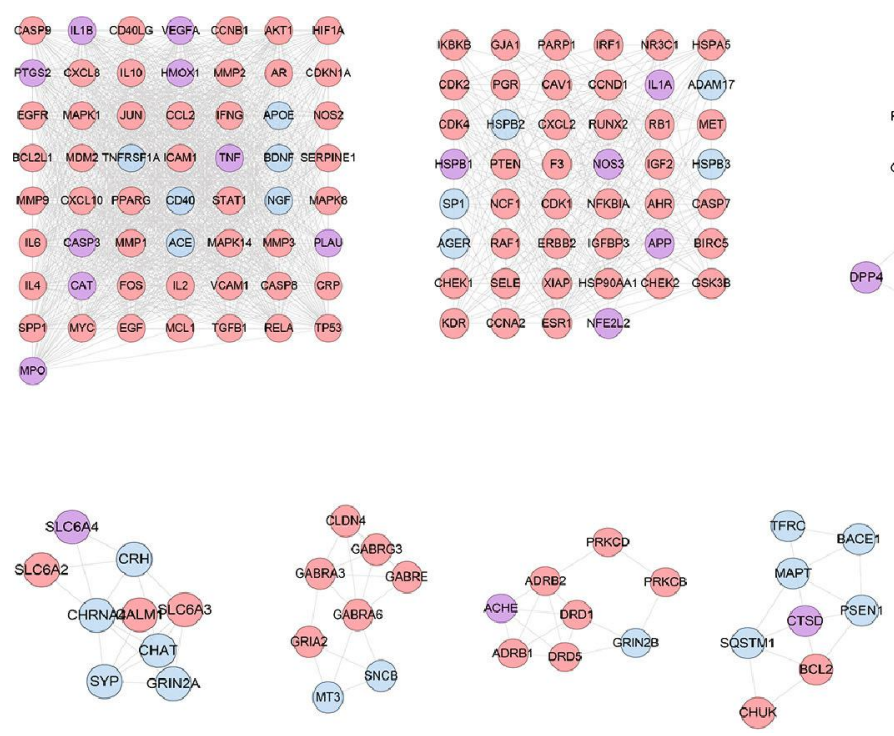
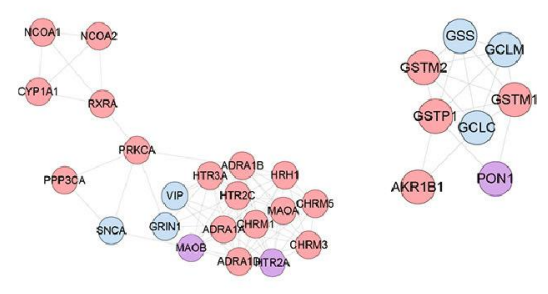

Figure 7: Clusters of FGWYD-VD PPI network (pink, blue, and purple circles stand for FGWYD targets, VD targets, and FGWYD-VD targets, respectively).

TCMSP. The VD-related genes with relevance score $>5.5$ were selected for sequence research. A total of $145 \mathrm{VD}$ genes were obtained from that database. Among those FGWYD targets, Morindae Officinalis Radix gets 34 potential targets, Arum Ternatum Thunb. gets 84 targets, Radix Rhei Et Rhizome gets 44 targets, Aconiti Lateralis Radix Praeparata gets
21 targets, Cinnamomi Ramulus gets 20 targets, Panax Ginseng C. A. Mey. gets 99 targets, Panax Notoginseng (Burk.) F. H. Chen Ex C. Chow gets 168 targets, Zingiber Officinale Roscoe gets 35 targets, Acoritataninowii Rhizoma gets 80 targets, and Epimrdii Herba gets 218 targets. There is overlap between the target set of FGWYD herbs and the VD gene set (Figure 3). 


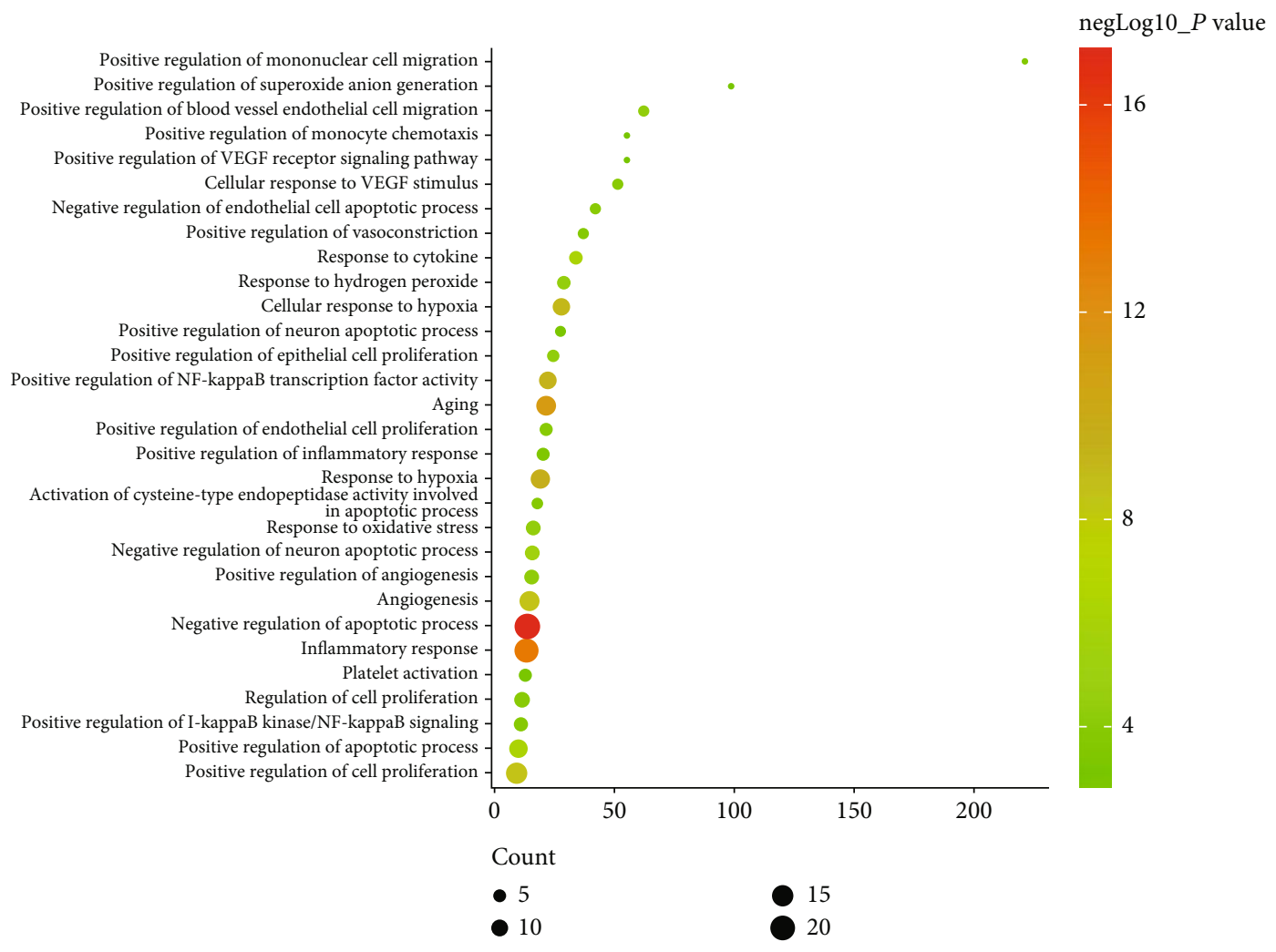

(a)

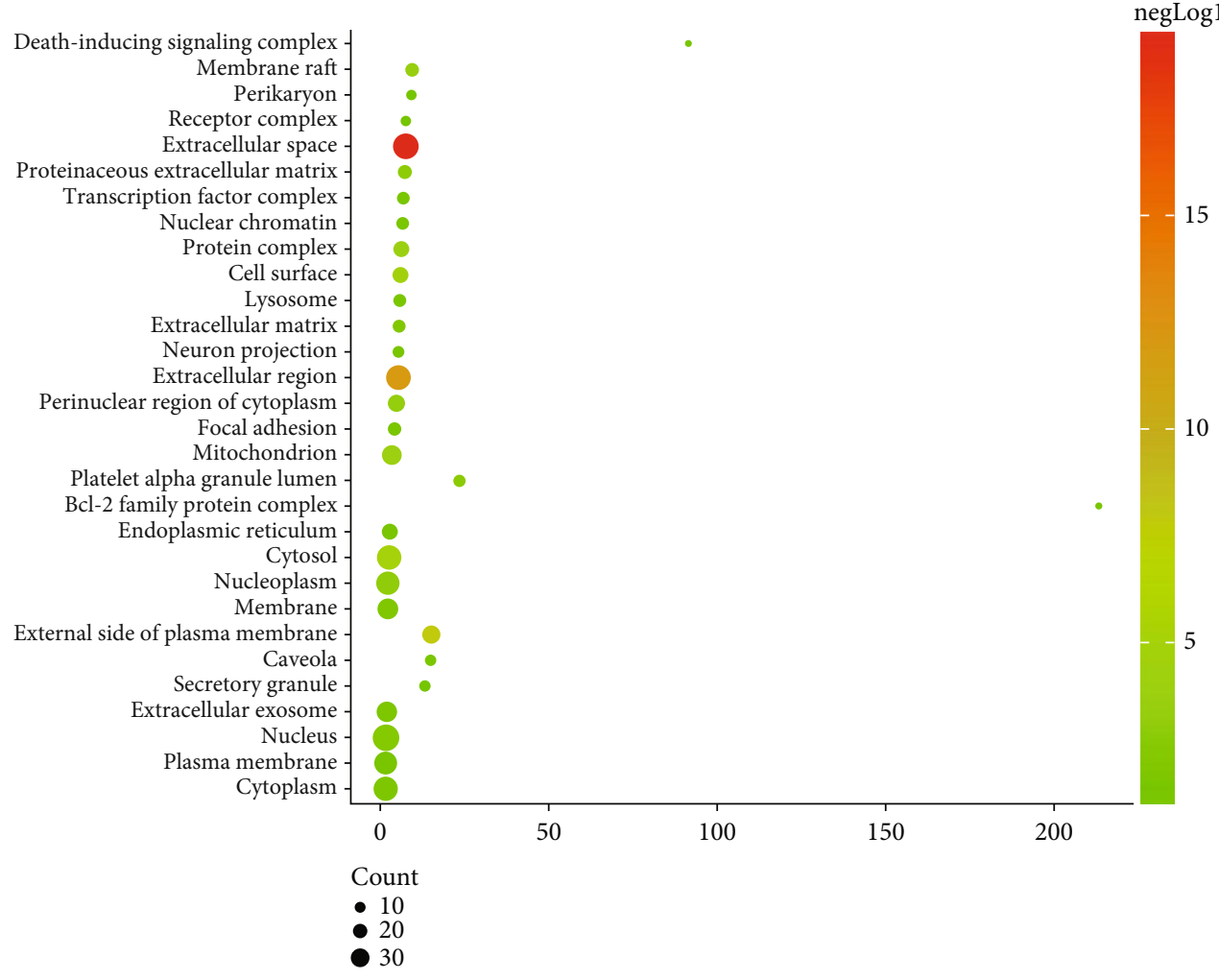

(b)

Figure 8: Continued. 


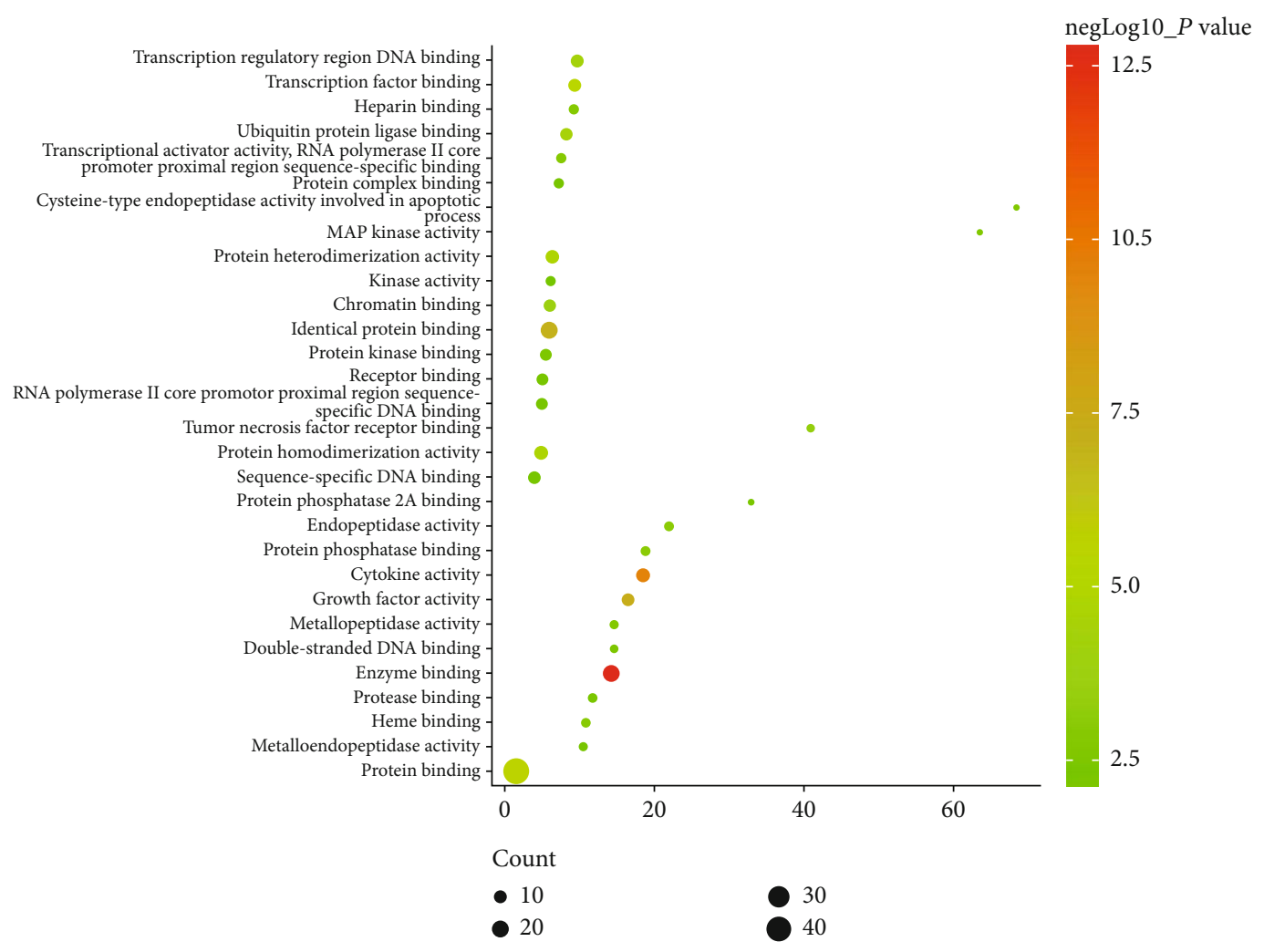

(c)

FiguRE 8: Bubble chart of cluster 1 ((a) biological processes; (b) cell components; (c) molecular function; $x$-axis stands for fold enrichment).

The FGWYD components and FGWYD targets were input into Cytoscape to construct component-target network of FGWYD. This network consists of 73 FGWYD component nodes, 245 target nodes and 964 edges (Figure 4). The nodes with higher degree was bigger in this network.

\subsection{FGWYD-VD PPI Network Analysis}

3.2.1. FGWYD-VD PPI Network Construct. The FGWYD targets, VD genes, and their PPI data were input into Cytoscape to construct the FGWYD-VD PPI network. This network is composed of 512 nodes (213 compound targets, $267 \mathrm{VP}$ genes, and 32 BHD-VP targets) and 8468 edges (Figure 3). The targets are arranged in descending order of degree. The top 10 targets in each target set are: (1) FGWYD target set: AKT1 (185 edges), IL6 (163 edges), TP53 (158 edges), EGFR (135 edges), MAPK1 (134 edges), MYC (130 edges), EGF (126 edges), MAPK8 (125 edges), JUN (125 edges), and FOS (120 edges); (2) VD target set: BDNF (104 edges), APOE (89 edges), NGF (88 edges), ACE (71 edges), SP1 (65 edges), TNFRSF1A (61 edges), GFAP (51 edges), CD40 (49 edges), MAPT (48 edges), and CLU (47 edges); and (3) FGWYD-VD target set: CASP3 (143 edges), TNF (141 edges), VEGFA (140 edges), APP (119 edges), PTGS2 (114 edges), CAT (107 edges), IL1B (99 edges), NOS3 (90 edges), HMOX1 (84 edges), and SOD1 (82 edges) (Figure 5). The primary enrichment analysis results are shown in Figure 6.
3.2.2. Biological Processes of FGWYD-VD PPI Network Construct. The FGWYD-VD PPI network was analyzed by MCODE and thirteen clusters were obtained (Table 5 and Figure 7). The targets in the clusters were input into DAVID to perform GO enrichment analysis, and got a lot of biological processes.

Cluster 1 is mainly related to apoptosis, inflammation, hypoxic response, angiogenesis, neuronal apoptosis, and oxidative stress. Cluster 2 is mainly related to angiogenesis, endoplasmic reticulum stress, inflammation, angiogenesis, and redox. Cluster 3 is mainly related to smooth muscle contraction, chemical synapses, synaptic transmission, and platelet activation. Cluster 4 is mainly related to synaptic transmission, neurotransmitter synthesis and catabolism, vasoconstriction, neuronal synaptic plasticity, and redox. Cluster 5 is mainly related to glutathione anabolism, oxidative stress, and mitochondrial depolarization. Cluster 6 is mainly related to neurotransmitter metabolism and synthesis. Cluster 7 is mainly related to chemical synaptic transmission, dopamine uptake and synaptic transmission, and hypoxia. Cluster 8 is mainly related to synaptic transmission and nerve impulse. Cluster 9 is mainly related to endoplasmic reticulum calcium homeostasis, negative regulation of neuronal apoptosis, and autophagy. Cluster 10 is mainly related to steroid metabolism. Cluster 11 is mainly related to coagulation, fibrinolysis, and platelet activation. Cluster 12 is mainly related to blood coagulation. Cluster 13 is mainly related to oxygen free radicals, synapse 


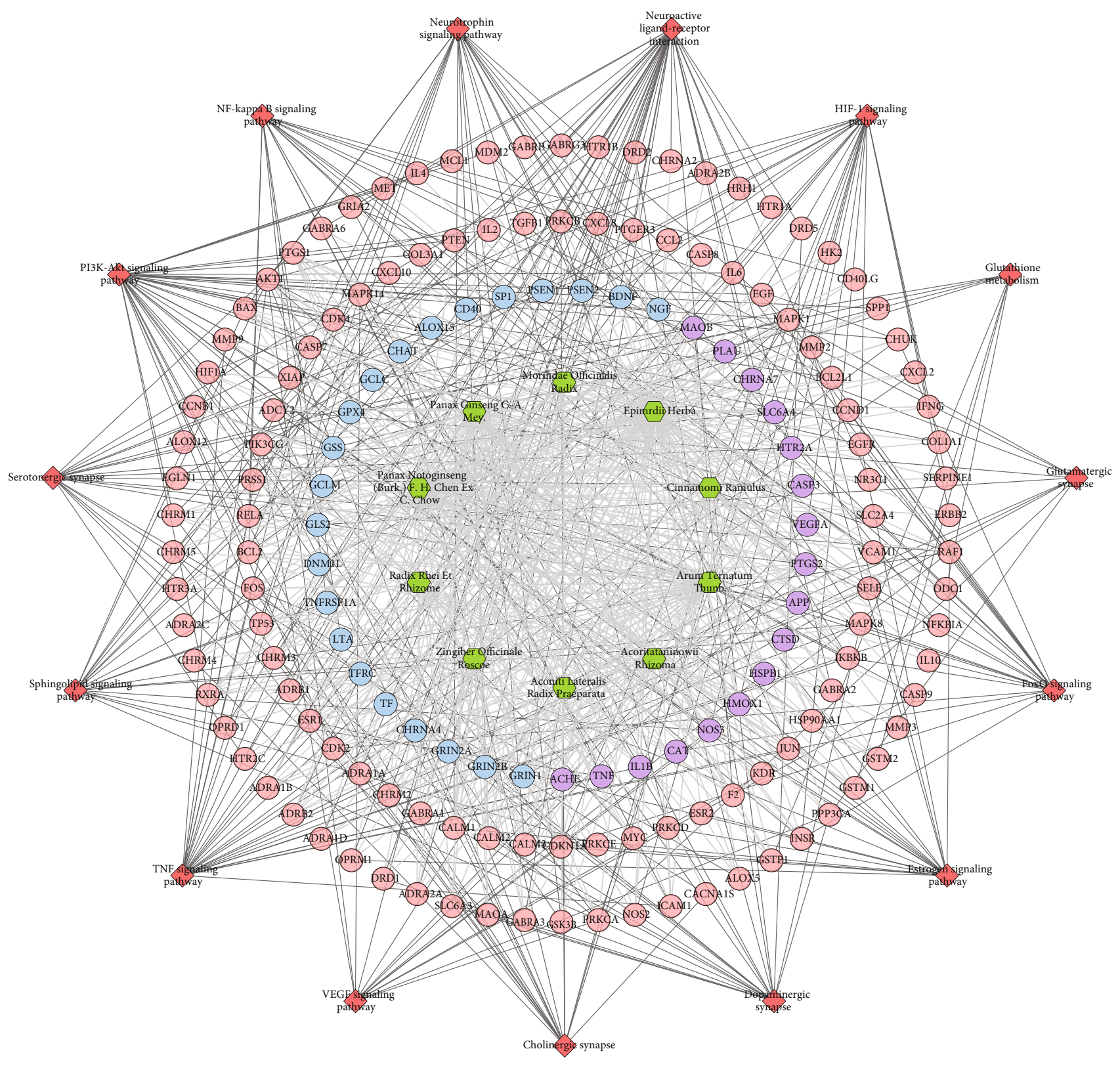

(a)

Figure 9: Continued. 


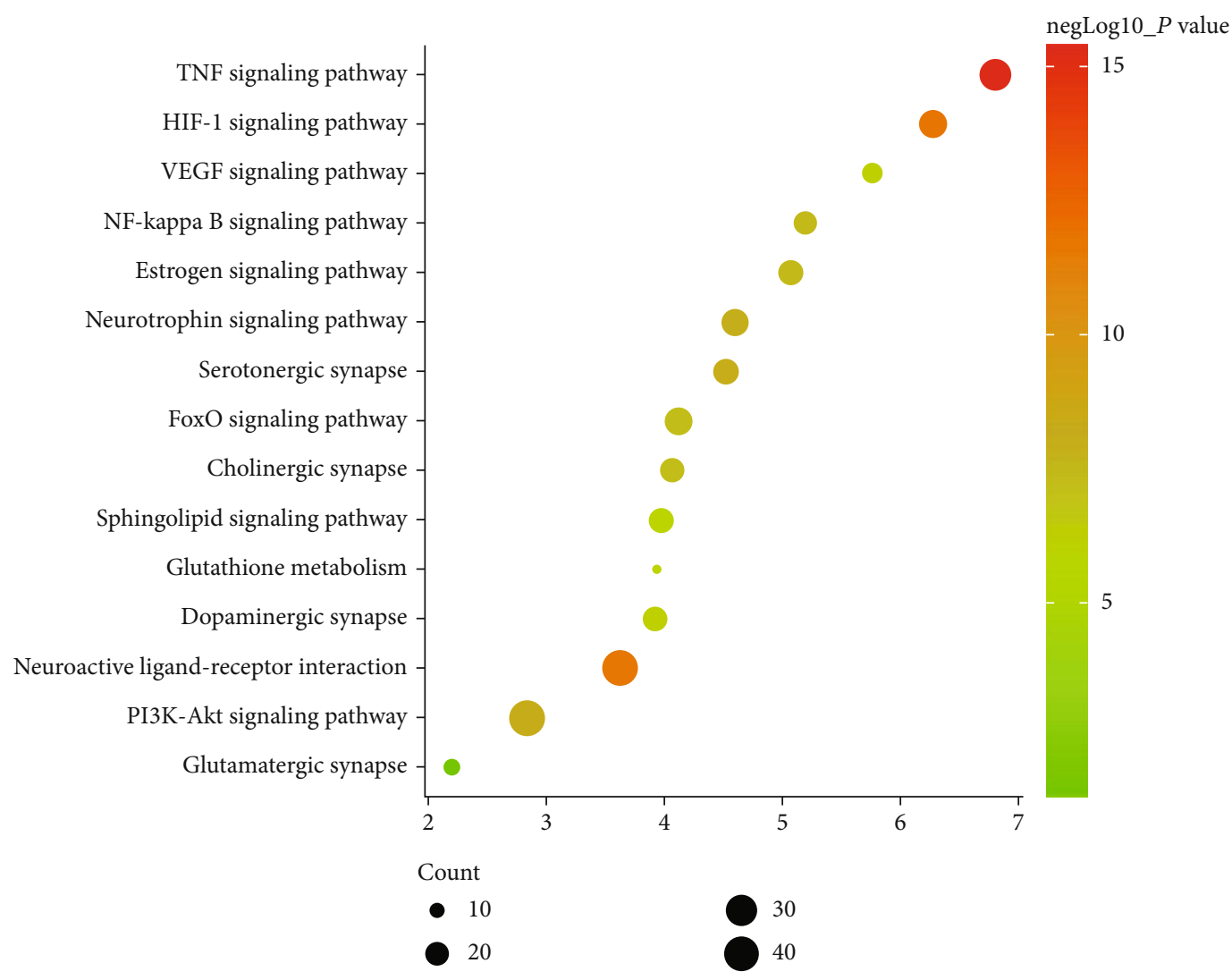

(b)

Figure 9: Signaling pathway of FGWYD-VD PPI network ((a) herb-target-signaling pathway network; blue, pink, and purple circles stand for the FGWYD target, VD genes, and the FGWYD-VD target, respectively; red diamonds stand for pathway; green hexagons stand for herb; (b) bubble chart of signaling pathway; $x$-axis stands for fold enrichment).

enhancement, and apoptosis (Table S3). The biological processes, cell components, and molecular function of cluster 1 is shown in Figure 8 as an example.

\subsubsection{Signaling Pathway of FGWYD-VD PPI Network. The} targets and genes in the FGWYD-VD PPI network was input into DAVID to perform pathway enrichment analysis, and it returned fifteen core VD-related pathways (Figure 9(a)). The top 10 signaling pathways are as follows: the TNF signaling pathway, the HIF-1 signaling pathway, neuroactive ligandreceptor interaction, the PI3K-Akt signaling pathway, the neurotrophin signaling pathway, the estrogen signaling pathway, the NF-kappa B signaling pathway, the FoxO signaling pathway, the serotonergic synapse, and the VEGF signaling pathway (Figure $9(\mathrm{~b})$ ). The details of the signaling pathway are shown in Table S4.

Current research shows that VD is currently the only preventable senile dementia, which is characterized by histopathological damage and progressive mental decline caused by hypoxic or hemorrhagic brain injury [29]. The hippocampus is an important structure for learning and memory, and it is extremely sensitive to cerebral ischemia and hypoxia. Cerebral ischemia and hypoxia can easily cause hippocampal neuron apoptosis and decrease learning and memory ability [30]. The currently generally accepted pathogenesis of VD includes cholinergic system dysfunction (acetylcholine deficiency or decreased choline acetyltransferase activity), neurosynaptic changes (decreased synaptic plasticity), excitatory amino acid toxicity damage, oxidative stress injury, and neuronal apoptosis [31-33]. New research shows that cerebrovascular changes may be involved in neurological dysfunction and cognitive impairment. Vascular endothelial dysfunction and neurovascular unit decoupling mediated by ischemia, hypoxia, oxidative stress, inflammation, and other factors can lead to neuronal damage or apoptosis, and ultimately cause cognitive impairment and neurodegenerative changes $[34,35]$. In summary, the etiology and pathogenesis of VD are complex, and searching and determining the key signal pathways or targets for the occurrence and development of VD are particularly important for the development of specific drugs. The application of systems biology and network pharmacology technology also has important hints for follow-up experimental research [36]. Based on the acquisition of FGWYD targets and VD-related genes, this study used bioinformatics techniques to analyze a total of 13 clusters and 14 signal pathways that may be involved in the prevention and treatment of VD by FGWYD and we found that Nrf2 (NFE2L2) and HO-1 (HMOX1) may play an important role in the treatment of VD by FGWYD (Figure 10). The integrated analysis of network biology modularity shows that the VD-related pathological biological modules mainly regulated by FGWYD are as follows: 


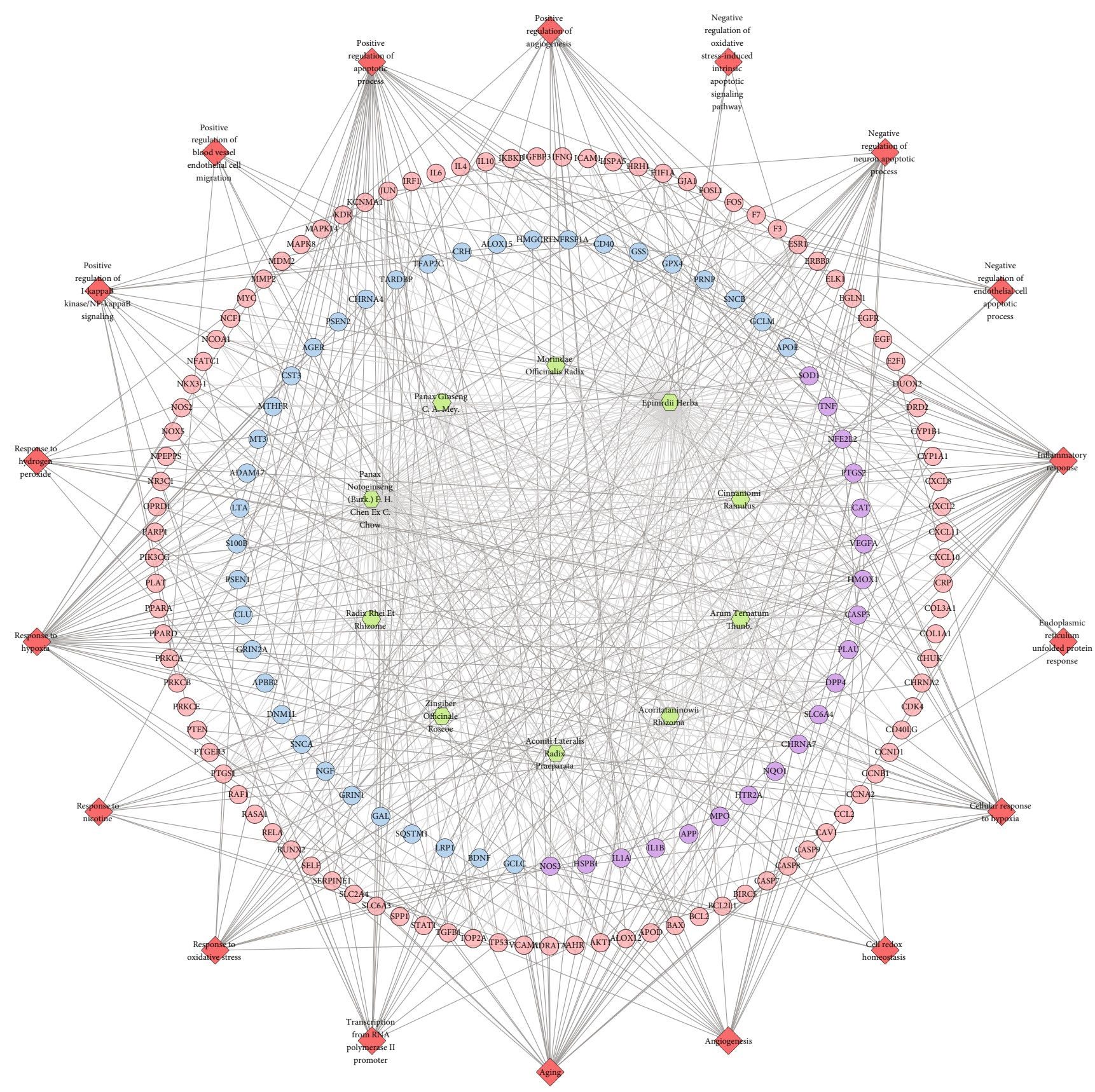

FIGURE 10: Herb-target-biological process network (blue, pink, and purple circles stand for the FGWYD target, VD genes, and the FGWYDVD target, respectively; red diamonds stand for the pathway; green hexagons stand for herb.)

inflammation module, oxidative stress, synaptic plasticity regulation module, neuronal apoptosis module, and angiogenesis module.

\subsection{The Results of Morris Water Maze Behavior Test}

3.3.1. Latent Period Results of Positioning Navigation Experiment. In the positioning navigation experiment, there was no significant difference in the incubation period of the rats in each group in the first two days, and there was no statistical difference. From the third day, compared with the model group, the latent period of the FGWYD medium-dose group, the high-dose group, and the sham operation group was significantly shortened $(P<0.01)$ (Figure 11).

3.3.2. Space Probe Experiment Results. In the space probe experiment, the effective residence time of the FGWYD medium- and high-dose groups was significantly prolonged, and there was no statistical difference compared with the sham operation group $(P>0.05)$. Compared with the model group, the effective residence time of other groups was significantly different $(P<0.01)$ (Figure 12). 


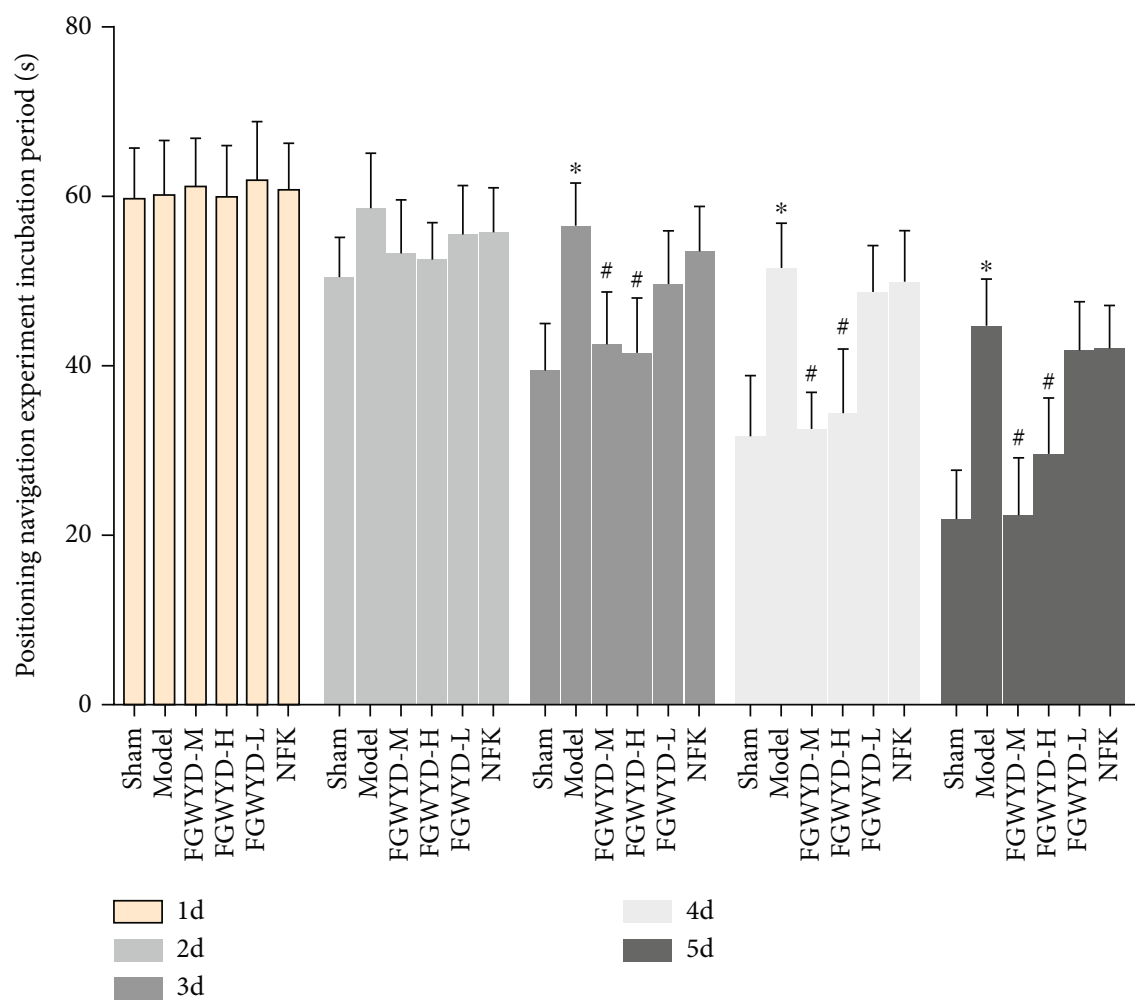

FIGURE 11: Latent period results of positioning navigation experiment (compared with sham operation group, ${ }^{*} P<0.01$; compared with the model group, $\left.{ }^{\#} P<0.01\right)$.
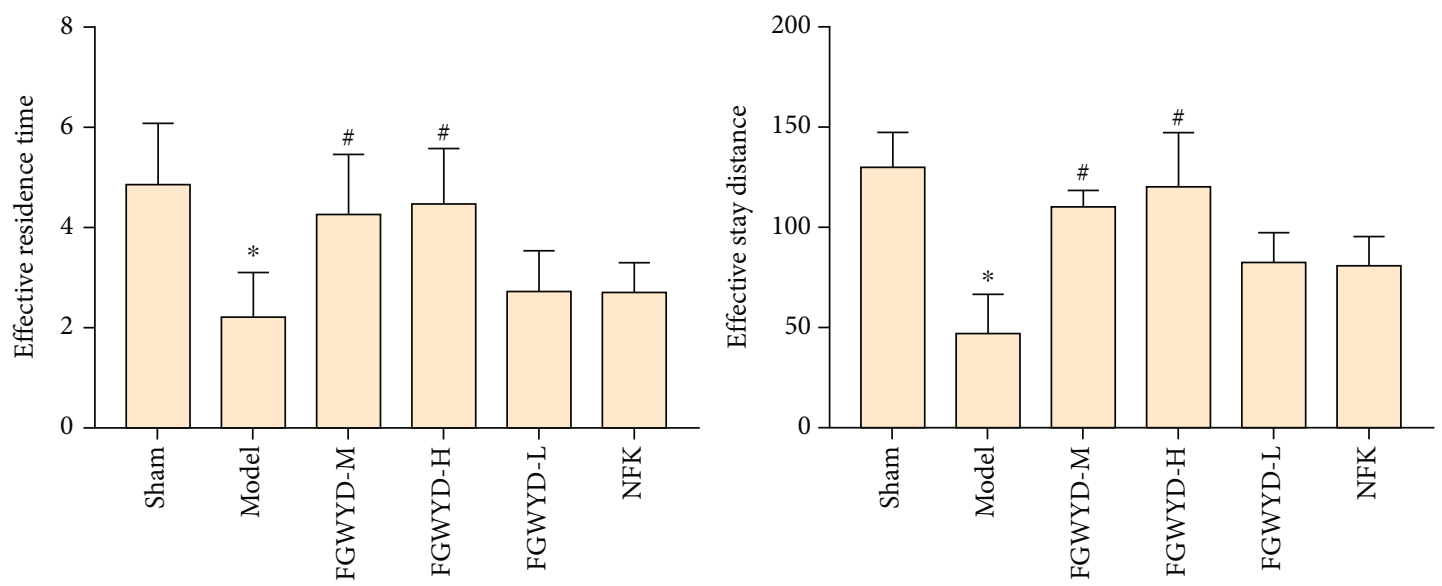

FIGURE 12: Space probe experiment results (compared with the sham operation group, ${ }^{*} P<0.01$; compared with the model group, ${ }^{\#} P<0.01$ ).

\subsection{Pathological Changes}

3.4.1. Sham Operation Group. In the sham operation group, the structure of the hippocampus is normal; the neurons in the hippocampus are tightly arranged, the cell structure is clear, the nucleus has no obvious pyknosis, the surrounding stroma has no obvious edema, the blood vessels have no obvious expansion, and the tissue has no obvious inflammatory cell infiltration (Figure 13(a)).

3.4.2. Model Group. In the model group, the structure of the hippocampus is abnormal. The number of neurons in the hippocampus is reduced, some neurons are arranged in dis- order, and the nuclei are constricted. Some neurons were pyknotic and deeply stained, there was no obvious edema in the surrounding interstitium, no obvious expansion of blood vessels, and no obvious inflammatory cell infiltration in the tissue (Figures 13(b) and 13(c)).

3.4.3. FGWYD High-Dose Group. In the FGWYD high-dose group, the structure of the hippocampus is normal. The cells are arranged neatly, there is no obvious pyknosis and deep staining, no obvious edema in the surrounding interstitium, no obvious expansion of blood vessels, and no obvious inflammatory cell infiltration in the tissue (Figure 13(d)). 


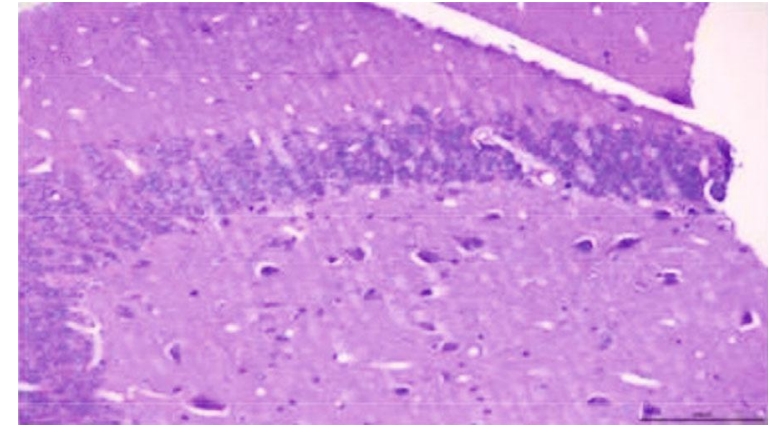

(a)

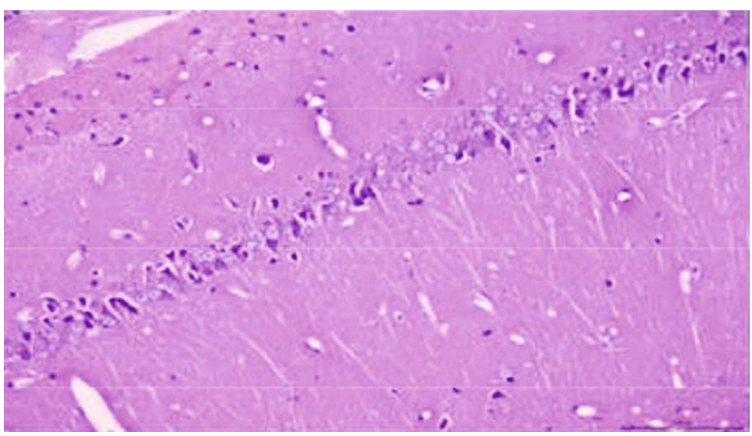

(c)

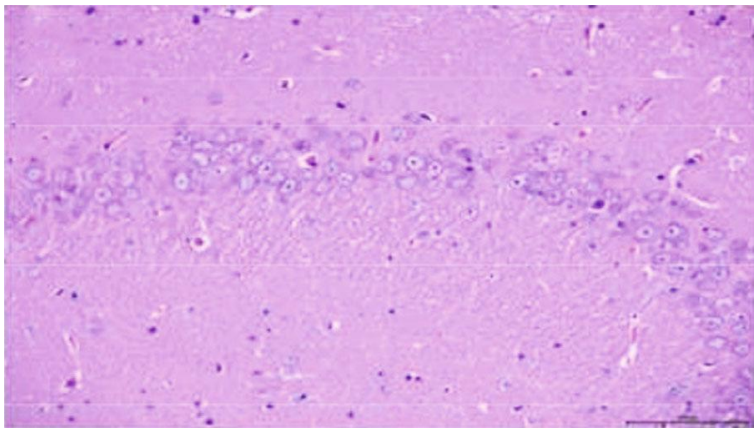

(e)

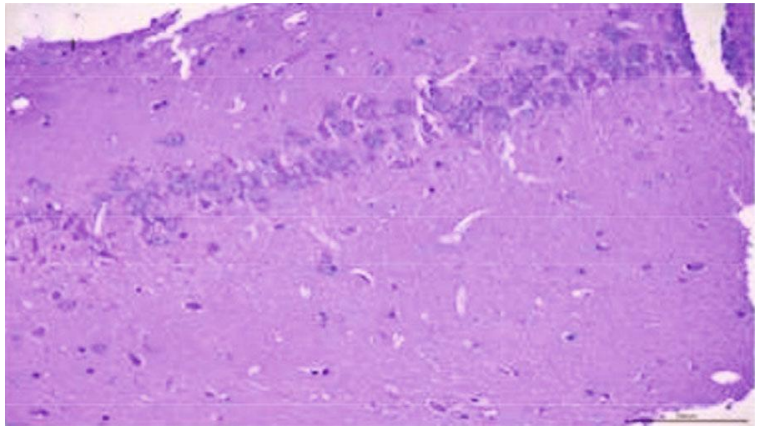

(b)

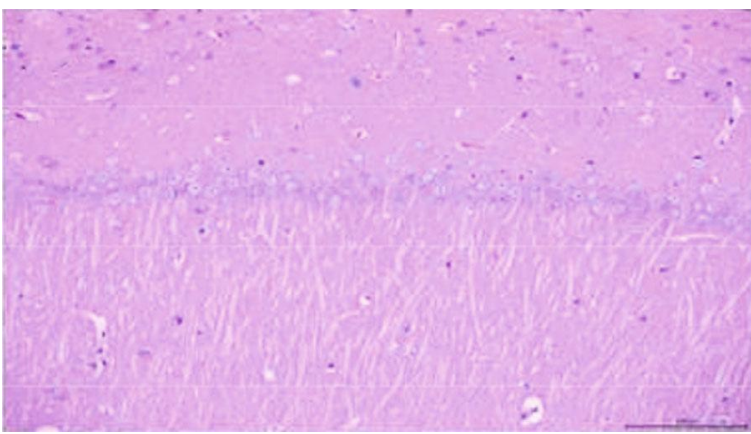

(d)

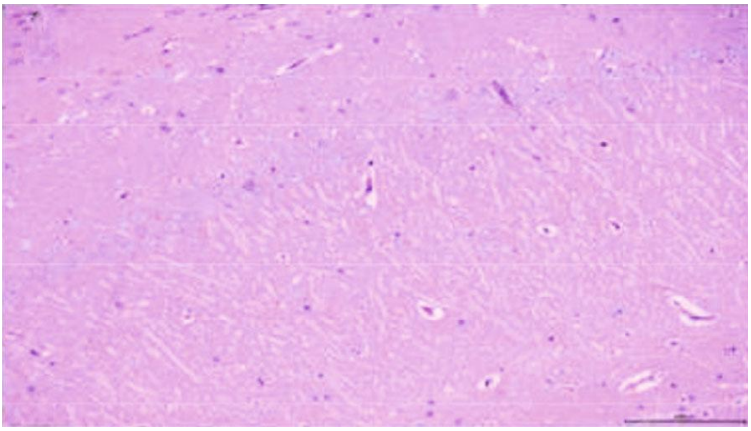

(f)

Figure 13: Pathological changes (HE staining, 200x; (a) sham operation group; (b) model group; (c) model group; (d) FGWYD high-dose group; (e) FGWYD low-dose group; (f) positive control group).

3.4.4. FGWYD Low-Dose Group. In the FGWYD low-dose group, the structure of the hippocampus is abnormal. It can be seen that individual neurons are pyknotic and deeply stained, the cell arrangement is basically neat, there is no obvious disorder, and the number of cells is not significantly reduced (Figure 13(e)).

3.4.5. Positive Control Group. In the positive control group, the structure of the hippocampus is abnormal. It can be seen that individual neurons are pyknotic and deeply stained; the cell arrangement is basically neat, without obvious disorder, and the number of cells is not significantly reduced (Figure 13(f)).

\subsection{Proteomics Results}

3.5.1. Differential Expression Protein Identification. Compared with the sham operation group, 23 proteins were upregulated and 17 proteins were downregulated in the 2- fold difference protein $(P<0.05)$ in the model group (Table 6). Compared with the model group, 16 proteins were upregulated and 10 proteins were downregulated in the FGWYD group with more than 2-fold differential protein $(P<0.05)$ (Table 7$)$.

3.5.2. Cluster Diagram of Differential Protein Expression Levels. In the control group, the expression of related genes in cluster 1 was downregulated, and the expression of related proteins in cluster 2 was upregulated. In the model group, the expression of related proteins in cluster1 was upregulated, and the expression of related proteins in cluster 2 was downregulated. In the FGWYD group, the expression of related proteins in cluster 1 was downregulated, and the expression of related proteins in cluster 2 was downregulated. The protein difference between the model group and the control group was obvious. After FGWYD, the protein expression recovery of the FGWYD group was similar to that of the control group. We can think that the modeling 
TABLE 6: The differential protein between the sham operation group and the model group.

\begin{tabular}{|c|c|c|}
\hline Protein TD & Description & Regulated \\
\hline F1LPB4 & Protein Akap9 & Up \\
\hline D4A050 & Protein Tbcld 32 & Up \\
\hline F1LUM5 & Tubulin alpha chain & Up \\
\hline Q63450 & Calcium/calmodulin-dependent protein kinase type 1 & $\mathrm{Up}$ \\
\hline A0A0G2K5Q2 & Crooked neck-like protein 1 & Up \\
\hline R9PXS3 & Transcription elongation factor, mitochondrial & Up \\
\hline A0A0G2K1C7 & Protein RGD1566386 & Up \\
\hline Q91ZW6 & Trimethyllysine dioxygenase, mitochondrial & Up \\
\hline F1M3H3 & Protein Frasl & Up \\
\hline P28470 & Calcineurin subunit B type 2 & Up \\
\hline M0RRJ7 & Complement $\mathrm{C} 3$ & Up \\
\hline Q5U2R9 & Protein Scfd2 & Up \\
\hline B0BNJ9 & RCG44002, isoform CRA a & Up \\
\hline Q5HZD9 & LOC100125377 protein & Up \\
\hline D4Al 17 & SID 1 transmembrane family member 1 & Up \\
\hline M0RCK7 & G-protein-coupled receptor 1 & Up \\
\hline Q3SWT7 & Nuclear receptor binding protein & Up \\
\hline F1LPTO & Gap junction protein & Up \\
\hline D3ZA65 & Protein Stk36 & Up \\
\hline M0R660 & Glyceraldehyde-3-phosphate dehydrogenase & Up \\
\hline F1LTH9 & Protein Wrn & Up \\
\hline D4A3T5 & Protein C 1 q13 & Up \\
\hline M0RBJ0 & Guanine nucleotide-binding protein subunit gamma & $\mathrm{Up}$ \\
\hline D3ZW33 & Protein Bach2 & Down \\
\hline D3ZTJ6 & Protein Tpcn2 & Down \\
\hline Q5M7T1 & Probable cytosolic iron-sulfur protein assembly protein CIAO1 & Down \\
\hline QSXIR9 & Ubiquitin-associated domain-containing protein 1 & Down \\
\hline Q510K8 & $28 \mathrm{~S}$ ribosomal protein S7, mitochondrial & Down \\
\hline Q91V33 & KHdomain-containing, RNA-binding signal transduction-associated protein 1 & Down \\
\hline M0R5Q3 & Protein Ranbp3 & Down \\
\hline A0A0G2K719 & Protein Ddx3x & Down \\
\hline D3ZE71 & Protein Faap24 & Down \\
\hline Q5XIMS & Protein CDV3 homolog & Down \\
\hline D3ZWV2 & Glyceraldehyde-3-phosphate dehydrogenase & Down \\
\hline D4A4U3 & Protein Mdpl & Down \\
\hline F1LWK7 & Protein Ablim 1 (fragment) & Down \\
\hline F1MlA6 & Protein T,OC681355 & Down \\
\hline D3ZEL3 & Protein TmcoSb & Down \\
\hline G3V6S6 & Protein Suv39h1l1 & Down \\
\hline A0A0G2K475 & Protein Brip 1 & Down \\
\hline
\end{tabular}

of the VD rat model group was successful, and the expression of related abnormal proteins was restored after FGWYD treatment (Figure 14).

3.5.3. Bioinformatics Analysis. The differentially expressed proteins of the sham operation/model group and the FGWYD/model group are combined and deduplicated. Then, they were imported into String, and the species was defined as "Rattus norvegicus," and other rat proteins related to these differentially expressed proteins and the PPI data were collected. Cytoscape was utilized to construct and analyze the network (Figure 15). This network consists of 448 nodes and 3137 edges (Table S5). The primary enrichment analysis results are shown in Figure 16. The clusters of this network are shown in Figure 17.

The proteins were input into DAVID to perform GO enrichment analysis and pathway enrichment analysis. The results showed that FGWYD may regulate VD-related 
TABLE 7: the differential protein between the FGWYD group and the model group.

\begin{tabular}{|c|c|c|}
\hline Protein ID & Description & Regulated \\
\hline A0A0G2K475 & Protein Brip 1 & Up \\
\hline Q5XIC2 & Evolutionarily conserved signaling intermediate in Toll pathway, mitochondrial & Up \\
\hline D3ZWV2 & Glyceraldehyde-3-phosphate dehydrogenase & Up \\
\hline G3V6S6 & Protein Suv39h111 & Up \\
\hline D3ZF71 & Protein Faap24 & Up \\
\hline D3ZFL3 & Protein TmcoSb & Up \\
\hline F1LWK7 & Protein Ablim 1 (fragment) & Up \\
\hline Q5M7T1 & Probable cytosolic iron-sulfur protein assembly protein CIAO1 & Up \\
\hline A0A096MK30 & Moesin & Up \\
\hline A0A0G2JVA8 & Protein Kb15 & Up \\
\hline P62329 & Thymosin beta- 4 & Up \\
\hline D4A050 & Protein Tbcl d 32 & Down \\
\hline Q5U2R9 & Protein Scfd2 & Down \\
\hline MORBJ7 & Complement C3 & Down \\
\hline D4A117 & SID 1 transmembrane family member 1 & Down \\
\hline A0A0G2K5Q2 & Crooked neck-like protein 1 & Down \\
\hline F1M3H3 & Protein Frasl & Down \\
\hline F1LUM5 & Tubulin alpha chain & Down \\
\hline F1LPB4 & Protein Akap9 & Down \\
\hline Q5XIG9 & Mitochondrial protein $18 \mathrm{kDa}$ OS & UP \\
\hline A0A0G2K475 & Protein Brip 1 & UP \\
\hline D4ACK7 & Protein Cnnm3 & UP \\
\hline P62329 & Thymosin beta- 4 & UP \\
\hline F1LNC3 & PH domain leucine-rich repeat protein phosphatase 1 & UP \\
\hline D4A050 & Protein Tbcl d32 & Down \\
\hline F1LPB4 & Protein Akap9 & Down \\
\hline
\end{tabular}

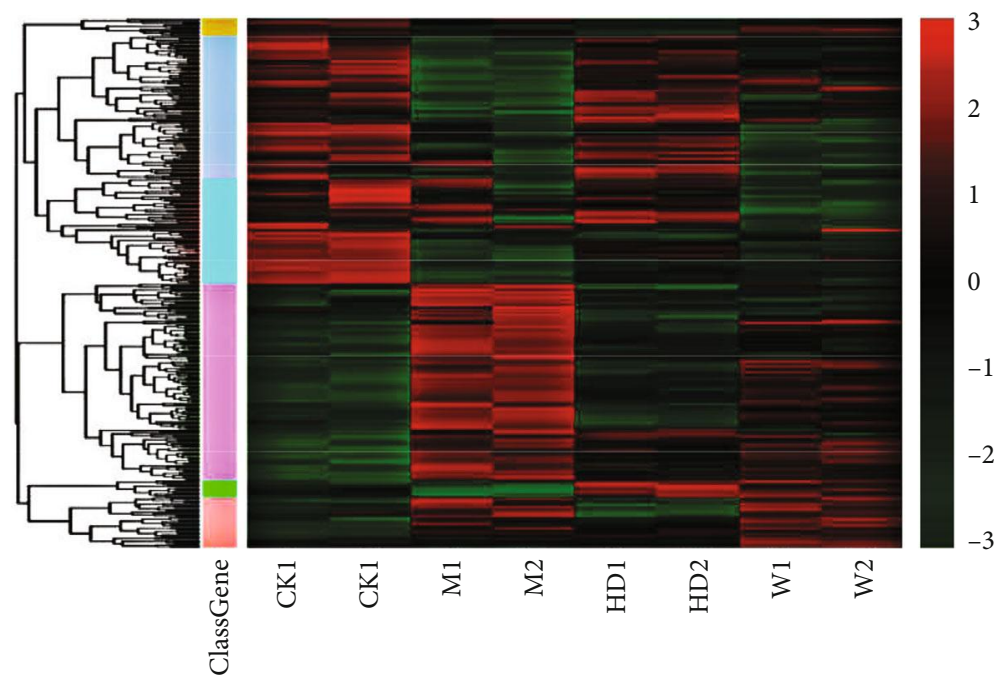

ClassGene

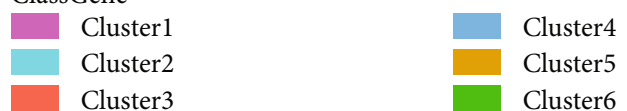

Figure 14: Cluster diagram of differential protein expression levels (CK1: control group 1; CK2: control group 2; M1: model group 1; M2: model group 2; HD1: FGWYD group 1; HD2: FGWYD group 2; W1: positive control group 1; W2: positive control group 2). 


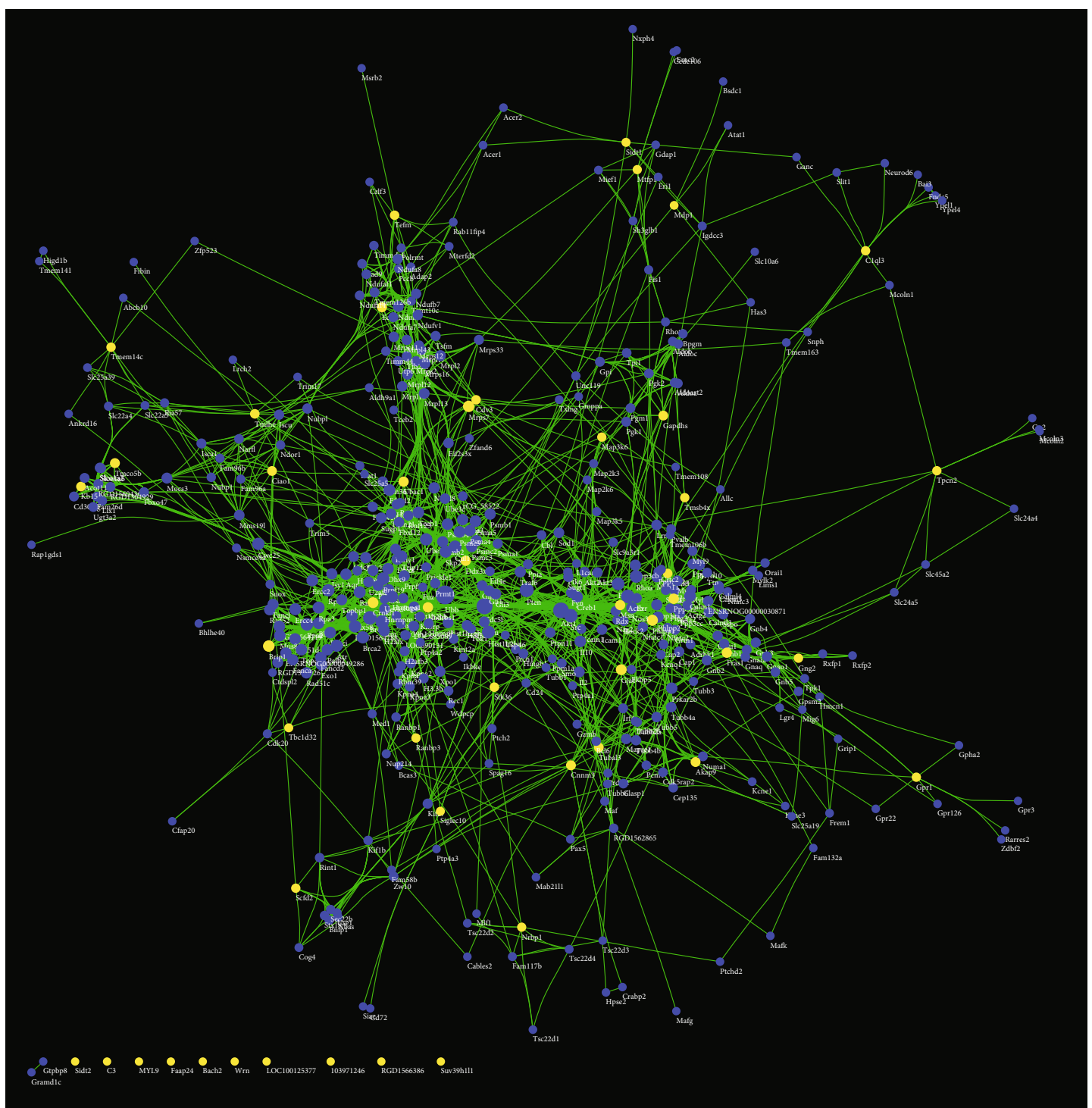

Figure 15: Differential expression protein-other rat protein PPI network (yellow circles stand for differential expression protein circle; blue circles stand for other rat protein circle).

biological processes and signaling pathways such as ironsulfur cluster assembly, calcineurin-NFAT signaling cascade, glycolytic process, cellular response to platelet-derived growth factor stimulus, smoothened signaling pathway involved in dorsal/ventral neural tube patterning, oxytocin signaling pathway, cGMP-PKG signaling pathway, dopaminergic synapse, circadian entrainment, glucagon signaling pathway, platelet activation, and B cell receptor signaling pathway (Figure 18 and Table S6).

In order to further illustrate the mechanism of FGWYD intervention in VD in animal models, this study combined isobaric tags for relative and absolute quantification (ITRAQ) with liquid chromatography-mass spectrometry to identify differential proteins and bioinformatics analysis in the hippocampus of VD rats. This provides new ideas for systematic research on the pathogenesis of $\mathrm{VD}$ and TCM treatment of VD. The biological function annotations of differentially expressed proteins show that FGWYD regulates the main biological processes of VD: iron metabolism (GO: 0016226), oxidative respiratory chain and other forms of mitochondrial energy metabolism (GO: 0032981 and GO: 0032543), and neuronal apoptosis (GO: 0070997). The signaling pathways of FGWYD for regulating VD mainly involve the following: nerve synapse remodeling and neurotransmitter synthesis and transmission, oxidative stress, calcium regulation signaling pathway, Alzheimer's disease, and neurotrophin signaling pathway).

\subsection{Effect of FGWYD on the Expression of Nrf2 Protein in VD Rats}

3.6.1. Nrf2 Protein Expression in the Nucleus. The Nrf2 protein content in the hippocampal nuclei of each group was statistically significantly different $(P<0.01)$; there was a 


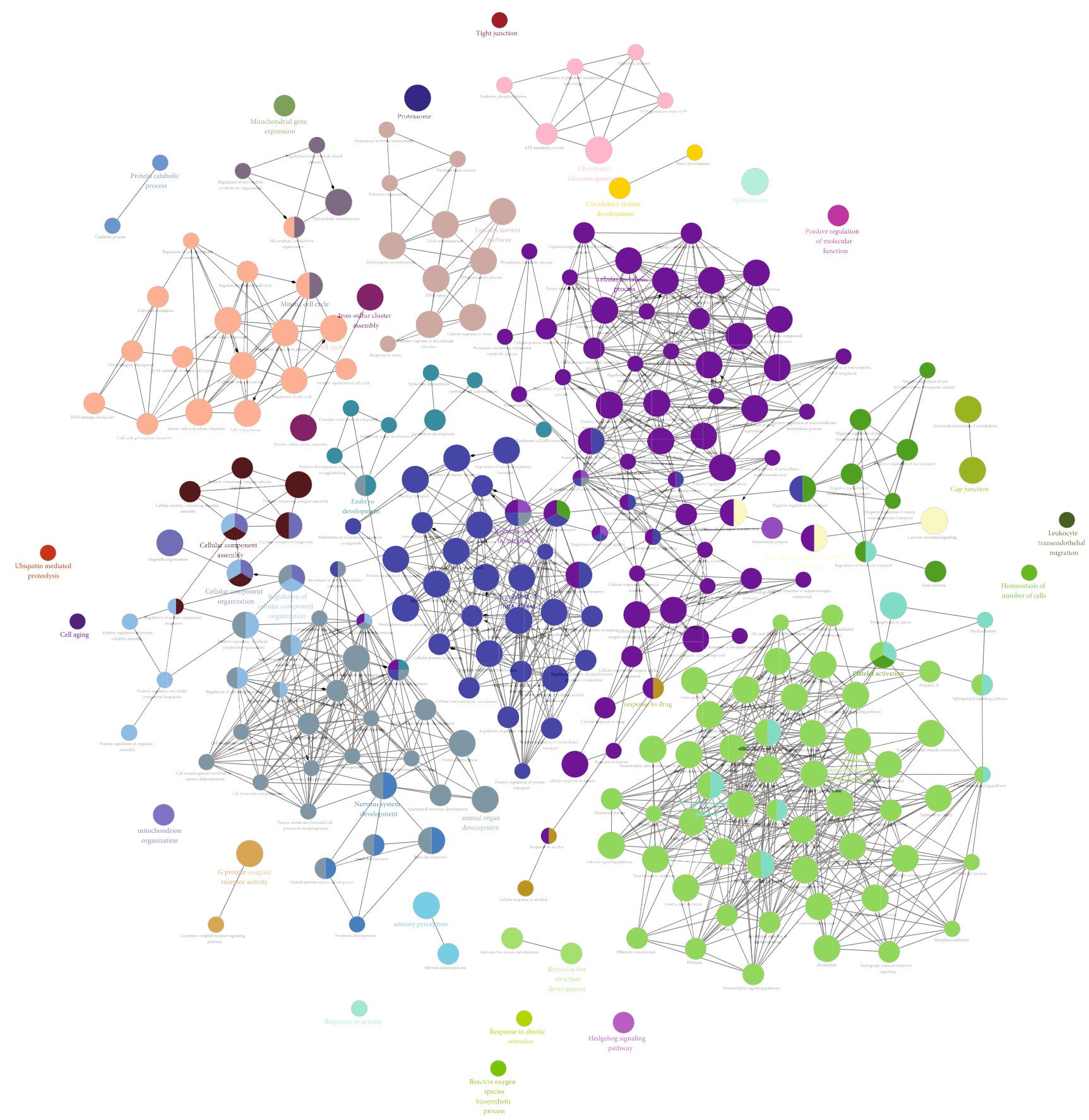

FIGURE 16: The primary enrichment results of differential expression protein-other rat protein PPI network.

statistical difference between the FGWYD low-dose group and the positive group $(P<0.05)$. After AS cerebral ischemia-reperfusion injury, the Nrf2 protein pathway in the rat hippocampal nucleus is activated, and the Nrf2 protein enters the nucleus from the cytoplasm, and its expression increases in the nucleus. The expression of the Nrf2 protein in the hippocampal nucleus of rats in the model group increased. After medication, the Nrf2 protein in the hippocampus of the rat increased, and the FGWYD medium- and high-dose groups were the most significant (Figure 19).
3.6.2. Cytoplasmic Nrf2 Protein Expression. The Nrf2 protein content in the hippocampal nuclei of each group was statistically significantly different $(P<0.01)$. Among them, there was a statistical difference between the FGWYD low-dose group and the positive group $(P<0.05)$. Experiments have shown that after AS cerebral ischemia-reperfusion injury, the Nrf2 protein pathway in the rat hippocampal nucleus is activated, and the Nrf2 protein enters the nucleus from the cytoplasm, and its expression in the cytoplasm decreases. The expression of the Nrf2 protein in the hippocampus cytoplasm of rats in the model group was reduced. After medication, 


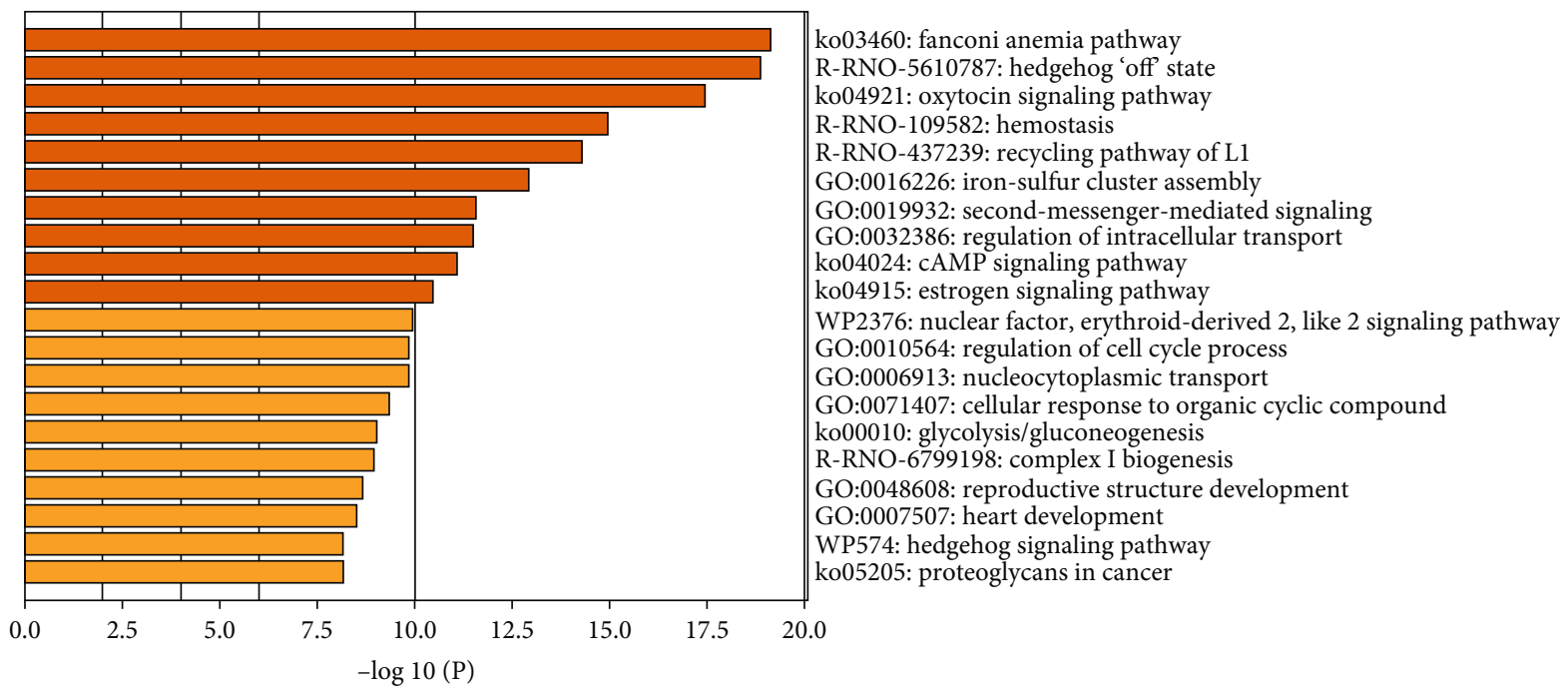

(a)
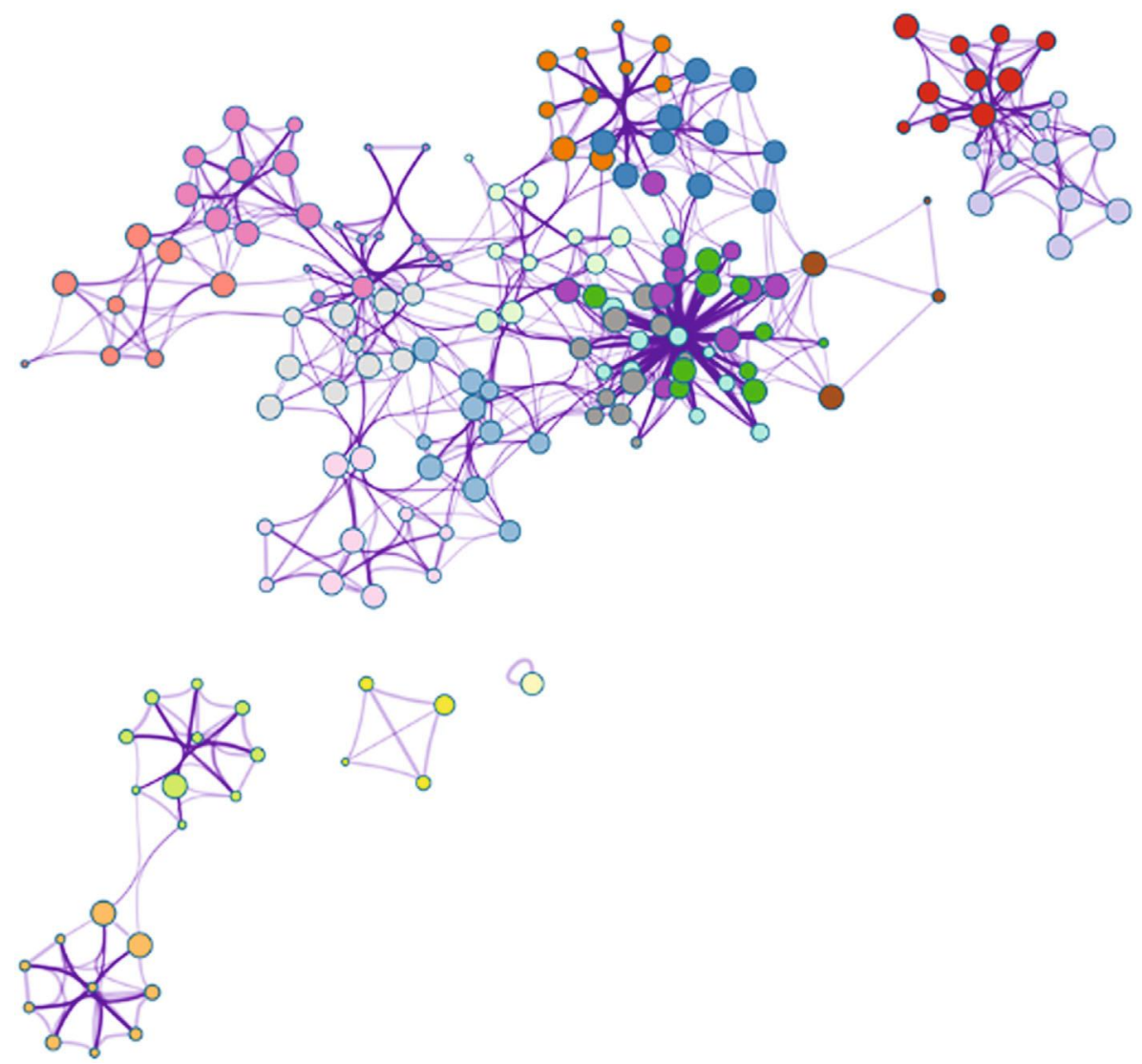

Fanconi anemia pathway Hedgehog 'off' state

Oxytocin signaling pathway

Hemostasis

Recycling pathway of L1

Iron-sulfur cluster assembly

Second-messenger-mediated signaling

Regulation of intracellular transport

cAMP signaling pathway

Estrogen signaling pathway

Nuclear factor, erythroid-derived 2, like 2 signaling pathway

Regulation of cell cycle process

Nucleocytoplasmic transport

Cellular response to organic cyclic compound

Glycolysis/gluconeogenesis

Complex I biogenesis

Reproductive structure development

Heart development

Hedgehog signaling pathway

Proteoglycans in cancer

(b)

FIgURE 17: Continued. 


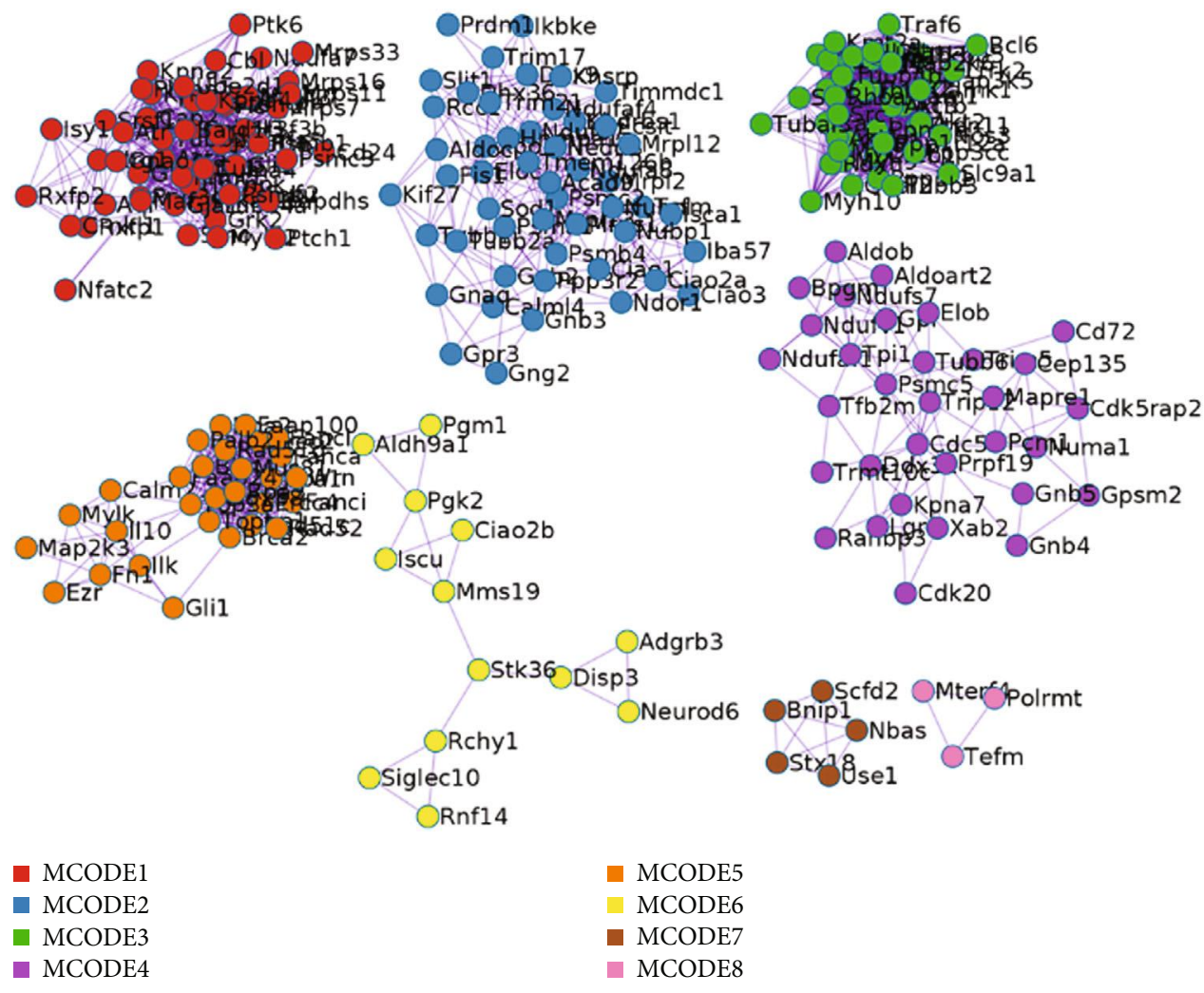

(c)

FIgURE 17: The metascape analysis results ((a) top enrichment results of proteomics proteins' PPI network; (b) PPI network colored by enrichment results; (c) clusters of PPI network).

most of the Nrf2 protein in the hippocampus of rats entered the nucleus from the cytoplasm, and the FGWYD mediumand high-dose groups were the most significant (Figure 19).

3.7. Effect of FGWYD on the Expression of HO-1 Protein in $V D$ Rats. Compared with the sham operation group, the HO-1 protein content in the hippocampus of each group was statistically significantly different $(P<0.01)$. Experiments have shown that after AS cerebral ischemiareperfusion injury, the Nrf2 protein pathway in the rat hippocampus nucleus is activated and the downstream protein HO- 1 is activated at the same time to increase its expression, and the FGWYD medium- and high-dose groups were the most significant (Figure 19).

3.8. The Results of EMSA. The extracted nucleoprotein and the Nrf2 probe formed an obvious binding zone. The addition of the Nrf2 antibody makes the binding band disappear, indicating that the complex contains Nrf2 protein. A 200fold concentration of a cold probe can inhibit this binding. If the possible Nrf2 binding element in the cold probe is mutated, the probe loses its binding ability, indicating that it is the Nt-2 binding element that binds to Nrf2 in the probe (Figure 20(a)).

The binding activity of Nrf2-ARE was not obvious in the sham operation group and the model group. The binding activity began to increase in the FGWYD low-, medium-, and high-dose groups and the positive group. Among them, the FGWYD high-dose group was the most significant, and the positive drug group was less (Figure 20(b)).

3.9. Effect of FGWYD on the Expression of HO-1 mRNA in VD Rats. Compared with the sham operation group, the expression of HO-1 mRNA in the hippocampus of each group was statistically significantly different $(P<0.01)$. Compared with the model group, the expression of $\mathrm{HO}-1$ mRNA in the FGWYD low-, medium-, and high-dose groups and the positive group was statistically different $(P<0.05)$ (Figure 21).

3.10. Effect of FGWYD on the MDA, SOD, GSH-Px, and LPO Contents and T-AOC in VD Rats. Compared with sham operation group, the MDA, SOD, GSH-Px, and LPO contents and T-AOC in the model group have statistical significance $(P<0.05)$. Compared with the model group, after drug (FGWYD or piracetam) intervention, the content of MDA and LPO decreased $(P<0.01)$, the content of SOD and GSH-Px increased $(P<0.01)$, and the T-AOC increased $(P<0.01)$ (Figure 22).

Based on the above comprehensive analysis of network pharmacology and proteomics, we chose oxidative stress as the research direction for further exploration of FGWYD intervention in VD. The main cause of VD is ischemic injury. In the state of ischemia and hypoxia, a large amount of oxygen free radicals will be produced in the brain, which can directly attack the unsaturated fatty acids in the 


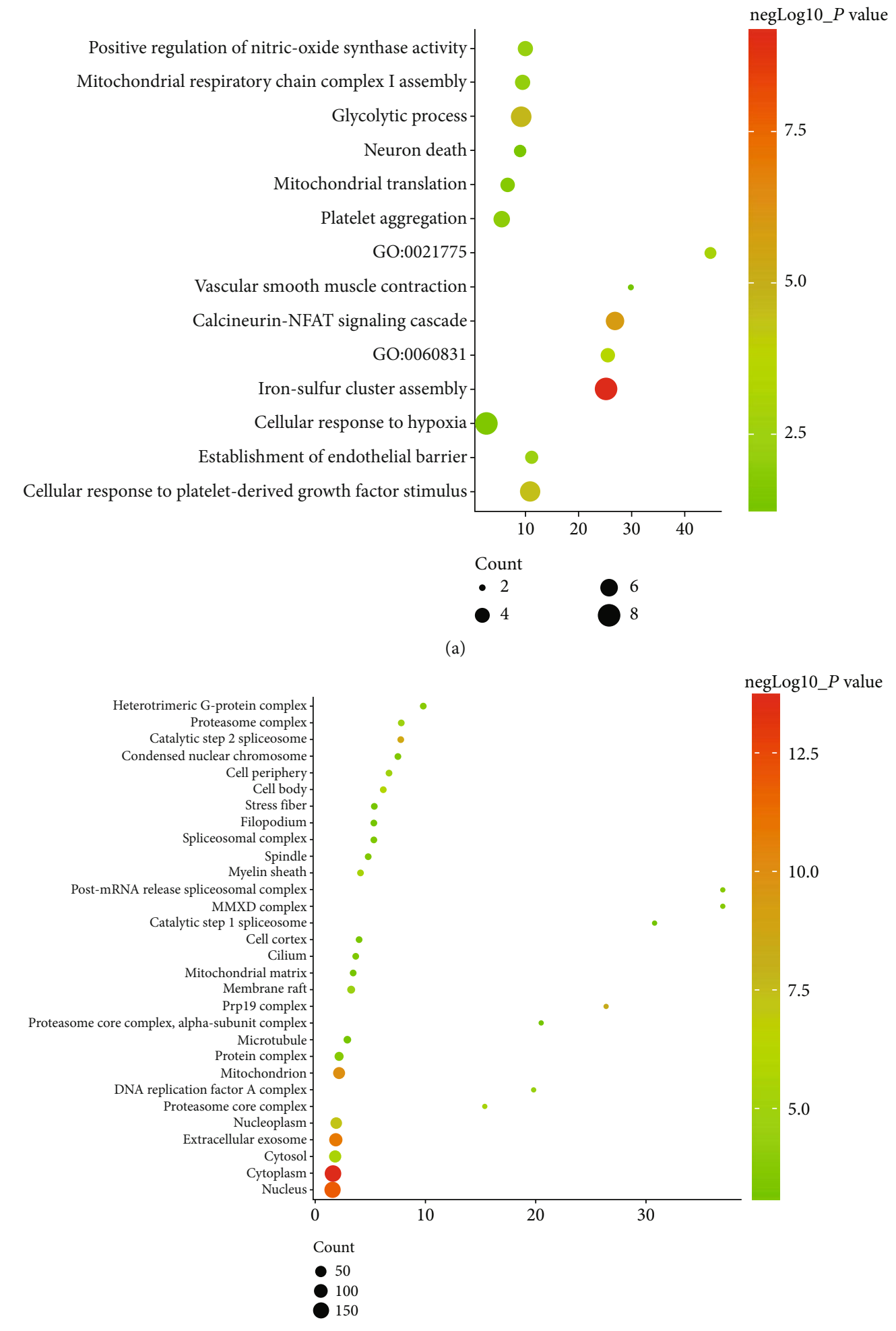

(b)

Figure 18: Continued. 


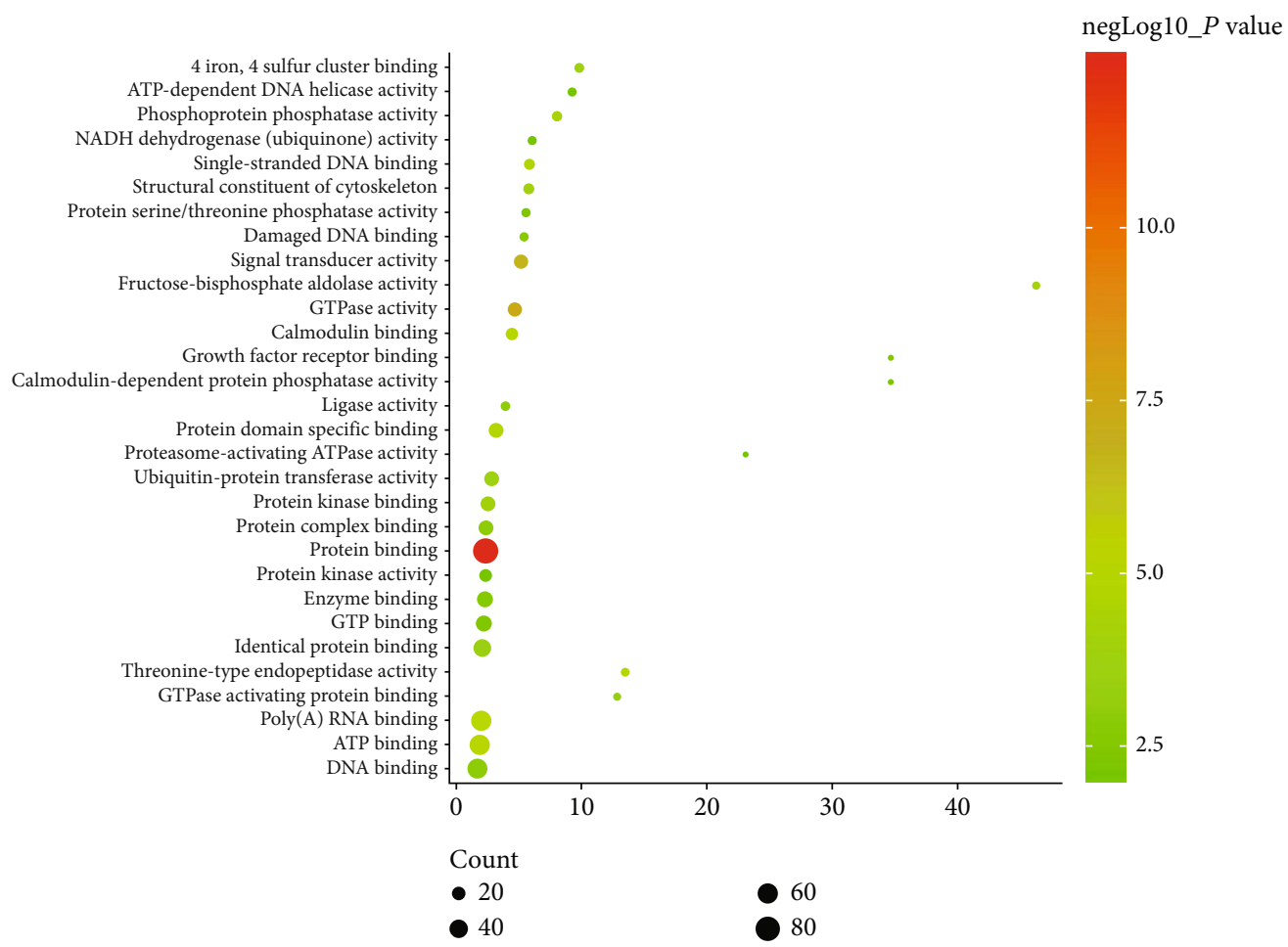

(c)

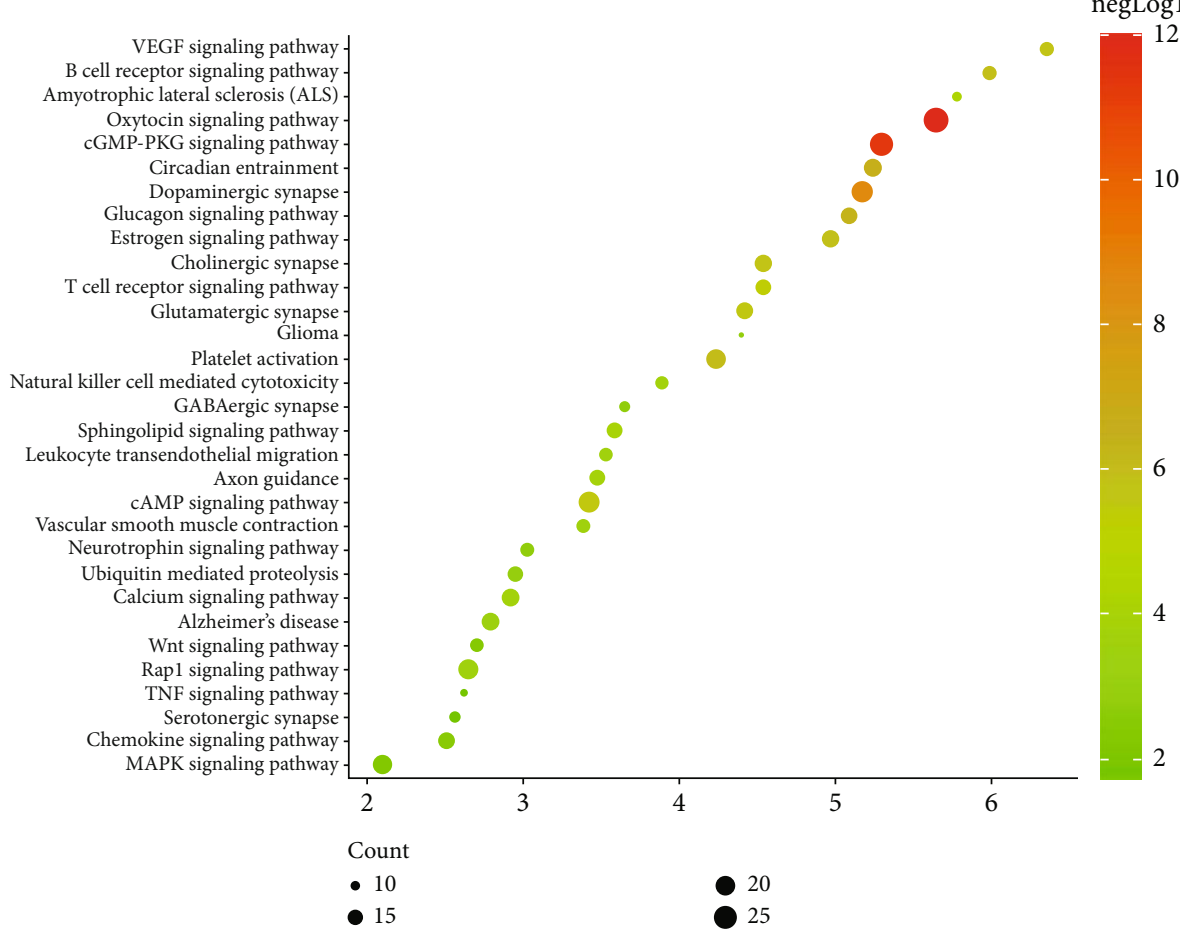

negLog10_P value

cin signaling pathway

Circadian entrainment

tominergic synapse

en signaling pathway

gnaling pathway

Platelet activatio

GABAergic synap

ansendothelial migratio Axon guidance okine signaling pathway

2

(d)

FIgURE 18: Bubble chart of bioinformatics analysis results ((a) biological processes; (b) cell components; (c) molecular function; (d) signaling pathways; $x$-axis stands for fold enrichment).

biomembrane phospholipids, causing damage to the brain nerve cells and dementia [37]. Nuclear factor erythroid 2related factor 2 (Nrf2) interacts with ARE to regulate the encoded antioxidant protein, forming the Nrf2-ARE path- way. This is a new type of antioxidant signaling pathway and the most important endogenous antioxidant stress pathway [38]. A large number of the downstream molecules that it regulates, including $\mathrm{HO}-1$, have multiple functions such as 


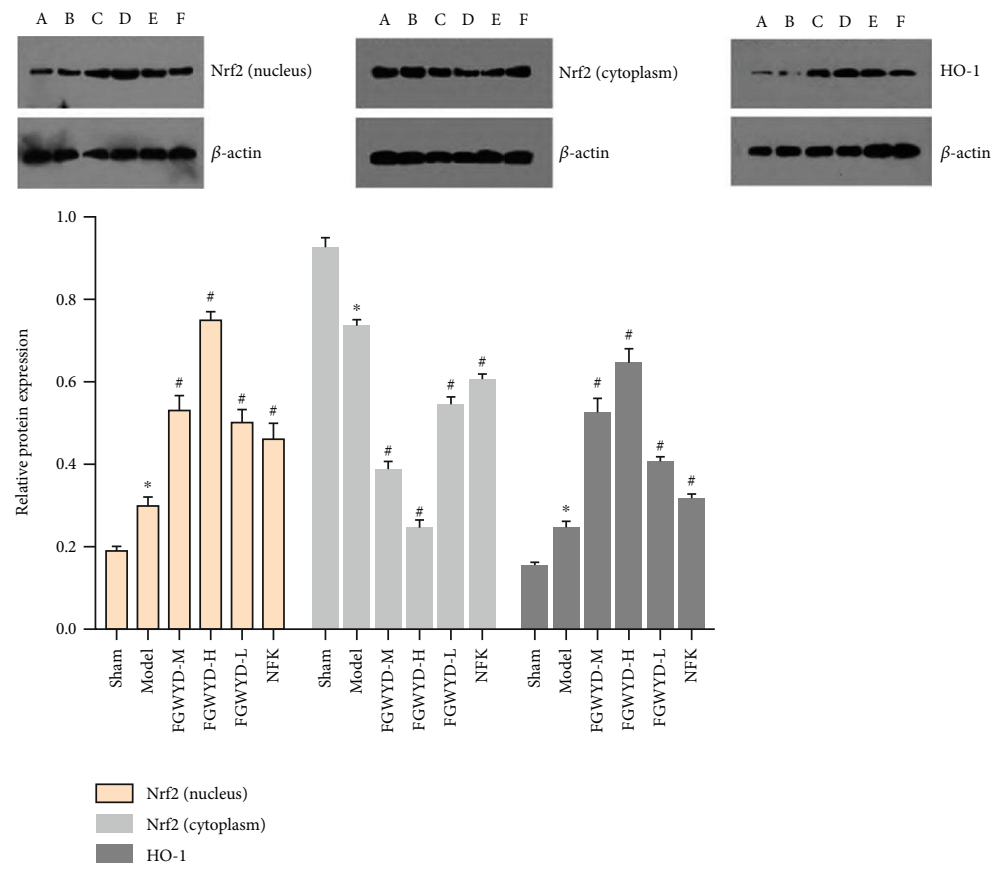

FIGURE 19: Effect of FGWYD on the expression of Nrf2 and HO-1 protein in VD rats ((A) sham operation group; (B) model group; (C) FGWYD medium-dose group; (D) FGWYD high-dose group; (E) FGWYD low-dose group; (F) positive control group; compared with sham operation group, ${ }^{*} P<0.01$; compared with model group, $\left.{ }^{\#} P<0.01\right)$.

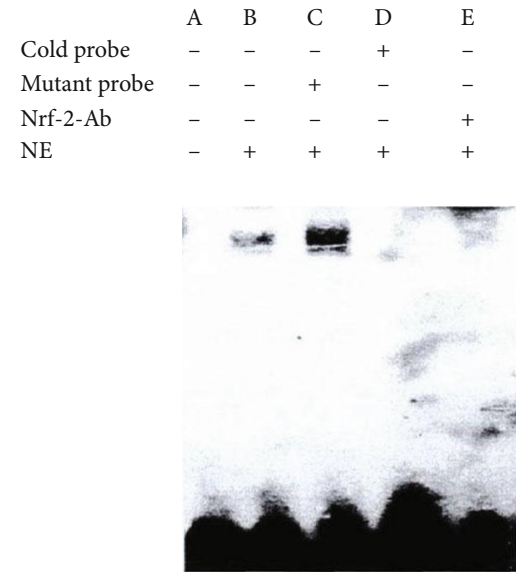

(a)

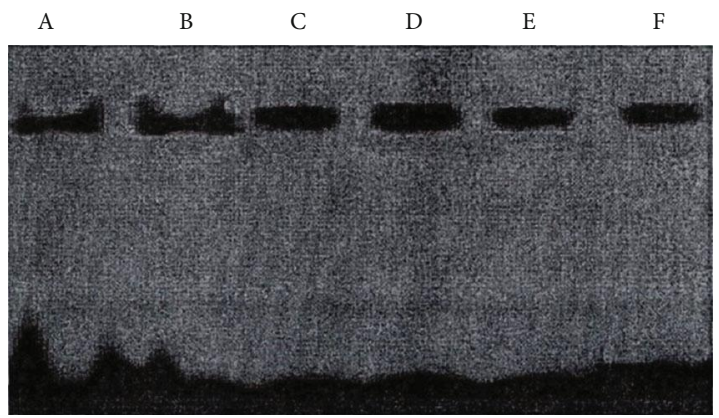

(b)

Figure 20: The results of EMSA. (a) Competitive assay. (b) EMSA (A: sham operation group; B: model group; C: FGWYD mediumdose group; D: FGWYD high-dose group; E: FGWYD low-dose group; F: positive control group).

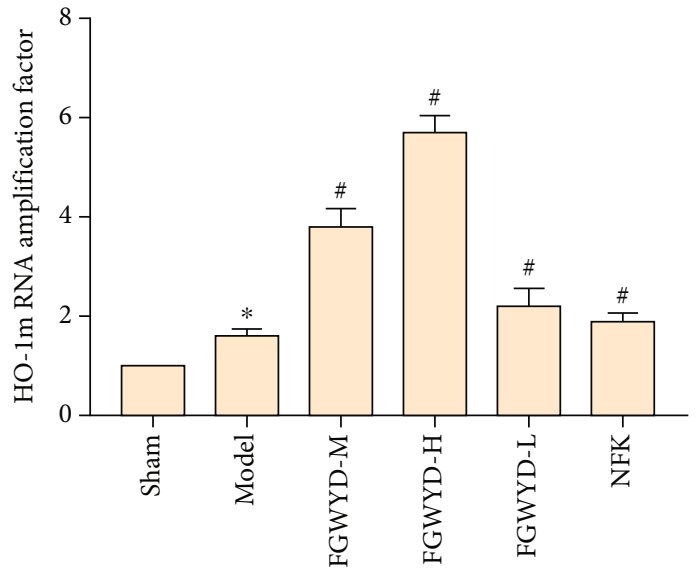

FIgURE 21: Effect of FGWYD on the expression of HO-1 mRNA in $\mathrm{VD}$ rats (compared with the sham operation group, ${ }^{*} P<0.01$; compared with the model group, ${ }^{\#} P<0.05$ ).

antioxidative stress, regulation of inflammatory damage, and antiapoptosis. Recent studies have shown that abnormal expression of Nrf2 or impaired transcriptional activity is closely related to the occurrence of ischemic encephalopathy [39]. The results of molecular docking showed that the core components of FGWYD can be stably combined with HMOX1 and NFE2L2, suggesting that FGWYD may interfere with VD by interfering with HMOX and NFE2L2 (Figure 23).

Free radical-mediated lipid peroxidation plays an important role in central nervous system diseases such as stroke, neurodegenerative diseases, mental disorders, and 

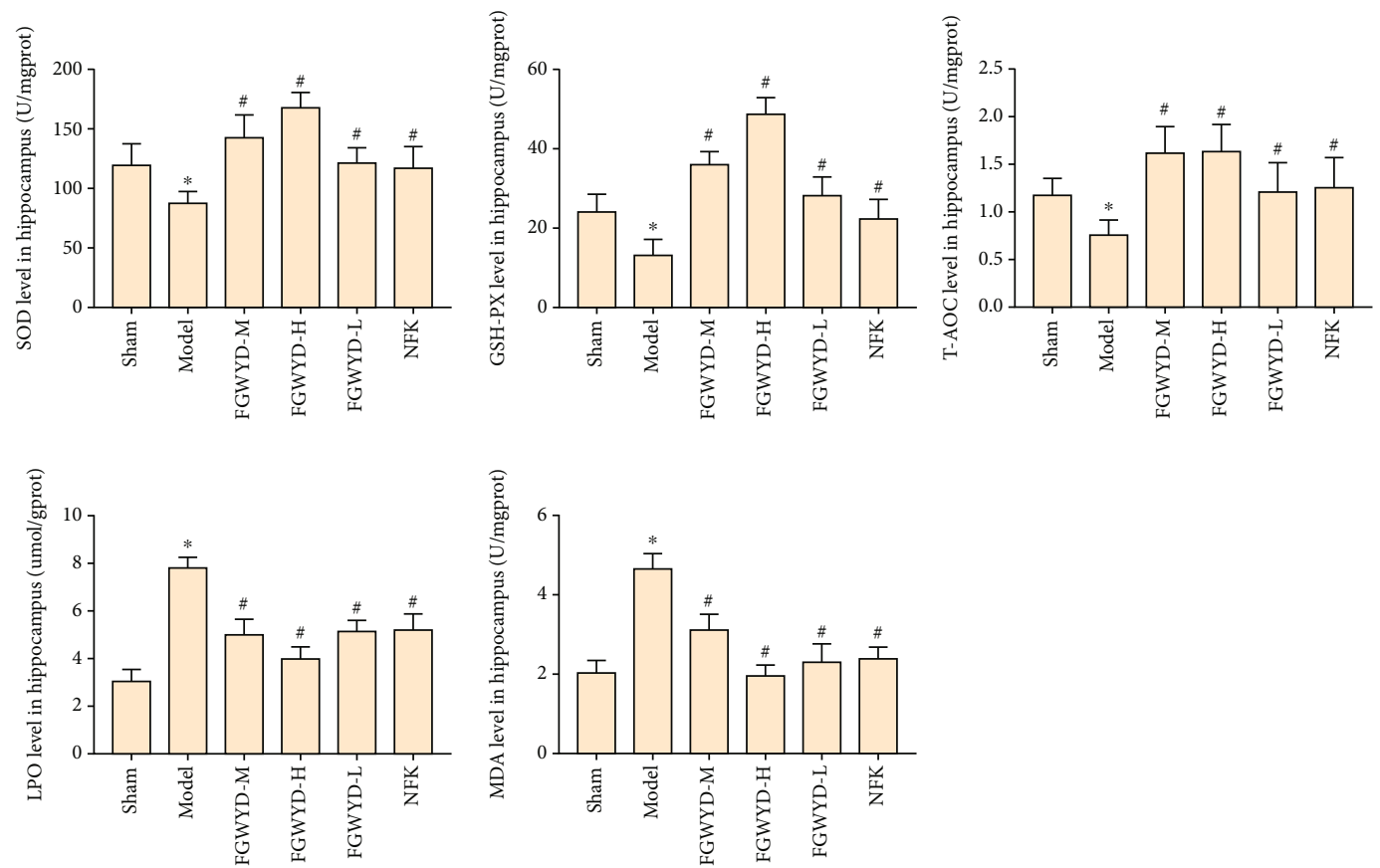

Figure 22: Effect of FGWYD on the MDA, SOD, GSH-Px, LPO, and T-AOC in VD rats (compared with sham operation group, ${ }^{*} P<0.05$; compared with model group, $\left.{ }^{\#} P<0.01\right)$.

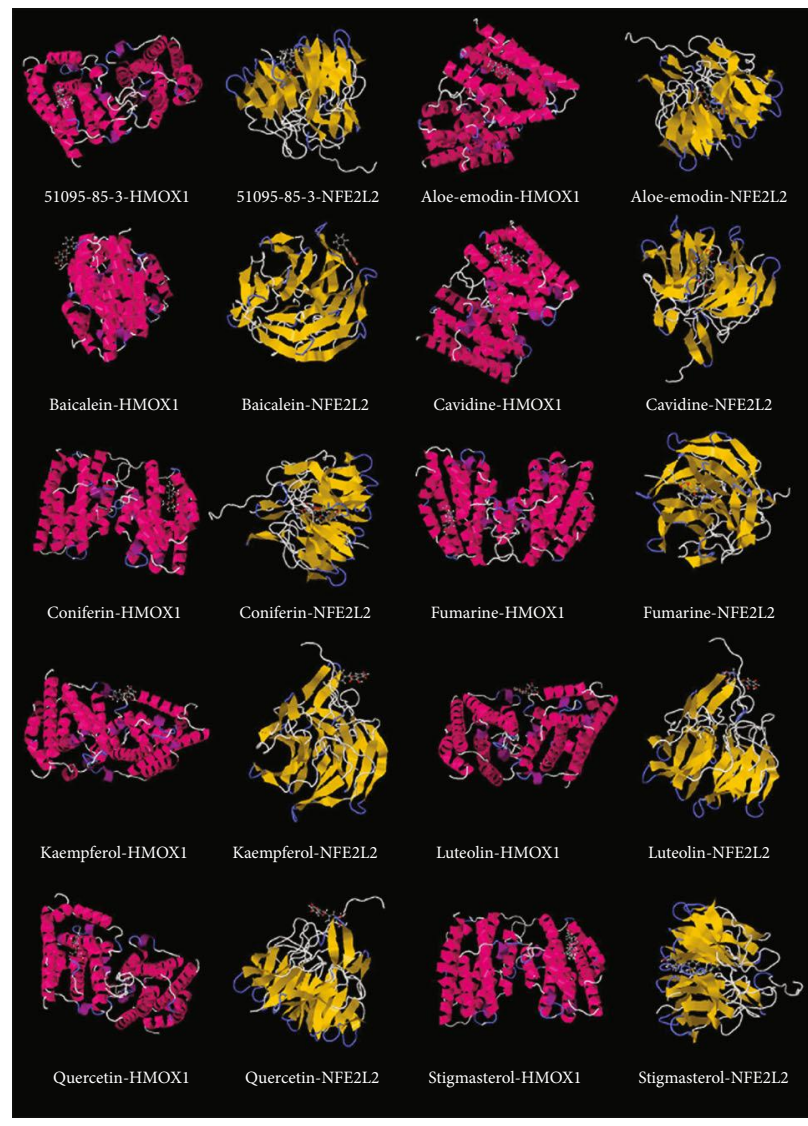

(a)

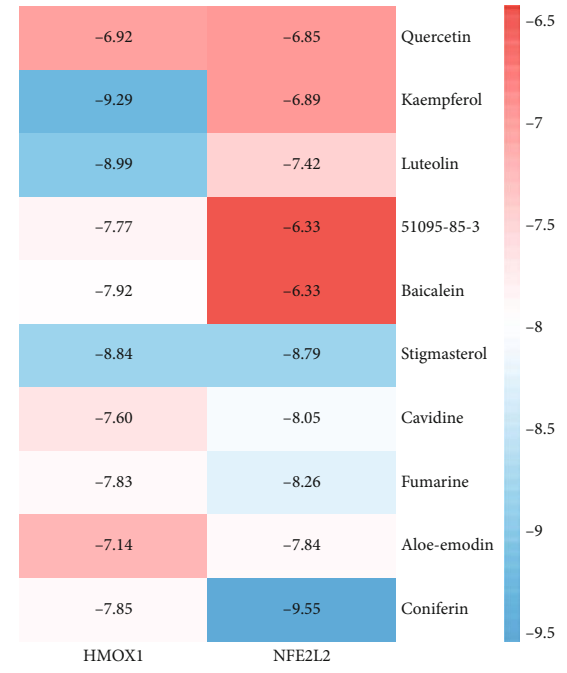

(b)

FIGURE 23: The results of molecular docking ((a) molecular docking mode diagram; (b) binding energy, kcal/mol). 
nervous system damage [40-43]. The damage caused by free radicals has been running through the process of nerve damage [44]. Free radicals are most likely to attack the double bonds of polyunsaturated acids in the cell membranes of brain cells. Free radicals will continue to damage proteins and nucleic acids, causing cell apoptosis. In nervous system diseases, due to various acute or chronic damages, free radicals will be produced, which will cause a series of cell apoptosis and the destruction of protein and DNA [45]. Current studies have found that the downstream antioxidant system HO-1 regulated by Nrf2-ARE does not contain much in the central nervous system. However, after Nrf2 is activated under prooxidant, inflammatory stimulus, and stress conditions, the expression of HO-1 in glial cells and astrocytes will increase. Studies in degenerative diseases of the central nervous system have shown that the content of HO-1 will also increase significantly, such as Parkinson's disease (PD), multiple sclerosis (MS), and amyotrophic lateral sclerosis (ALS) [46]. As the reduced glutathione contained in brain cells is low, brain cells are more susceptible to oxidative damage [47]. Enhancing the activity of endogenous antioxidant enzymes (such as CAT, GPxs, and SOD) after cerebral ischemia can reduce brain tissue damage $[48,49]$.

Our study established a rat model of VD caused by AS and used FGWYD for treatment. The results of the study show that FGWYD can activate the Nrf2-ARE pathway to transfer Nrf2 from the cytoplasm to the nucleus and increase its expression in the nucleus. At the same time, Nrf2 is phosphorylated and moved to the nucleus to induce the expression of the HO- 1 gene. HO-1 is an important part of brain cells against stress and oxidative damage. It can protect cells from oxidative stress and damage caused by foreign harmful substances.

\section{Conclusion}

The mechanism of FGWYD in the treatment of VD may be related to inflammation, oxidative stress, angiogenesis, and neuronal apoptosis.

\section{Data Availability}

The data used to support the findings of this study are included within the article and the supplementary information files.

\section{Disclosure}

Kailin Yang, Liuting Zeng, Anqi Ge, Chuandong Cao, Haiyan Zhang, and Tingting Bao should be considered joint first authors.

\section{Conflicts of Interest}

We declare no competing interests.

\section{Authors' Contributions}

Kailin Yang, Liuting Zeng, Anqi Ge, Chuandong Cao, Haiyan Zhang, and Jinwen Ge are responsible for the study concept and design. Kailin Yang, Liuting Zeng, Anqi Ge, and Yaqiao Yi are responsible for data analysis and interpretation in the chemoinformatics part. Chuandong Cao and Haiyan Zhang are responsible for data analysis and interpretation in experiments. Kailin Yang, Liuting Zeng, Anqi Ge, Chuandong Cao, and Haiyan Zhang drafted the paper. Jinwen Ge supervised the study. All authors participated in the analysis and interpretation of data and approved the final paper.

\section{Acknowledgments}

This work was supported by the National Natural Science Foundation of China (81774174).

\section{Supplementary Materials}

Supplementary 1. Table S1: potential targets for FGWYD.

Supplementary 2. Table S2: VD genes.

Supplementary 3. Table S3: biological processes of the FGWYD-VD PPI network.

Supplementary 4. Table S4: signaling pathway of the FGWYD-VD PPI network.

Supplementary 5. Table S5: proteomics data and other rat proteins.

Supplementary 6. Table S6: bioinformatics analysis of differential expression of the protein-other rat protein PPI network.

Supplementary 7. Figure S1: the results of HPLC: (a) reference solution; (b) FGWYD solution.

\section{References}

[1] O. Uwagbai and V. B. Kalish, “"Vascular dementia.” 2020 Mar 24," in StatPearls, StatPearls Publishing, Treasure Island (FL), 2020.

[2] L. L. Smits, A. C. van Harten, Y. A. Pijnenburg et al., "Trajectories of cognitive decline in different types of dementia," Psychological Medicine, vol. 45, no. 5, pp. 1051-1059, 2015.

[3] M. A. O. Santos, L. S. Bezerra, C. D. C. Correia, and I. S. Bruscky, "Neuropsychiatric symptoms in vascular dementia: epidemiologic and clinical aspects," Dement Neuropsychol., vol. 12, no. 1, pp. 40-44, 2018.

[4] L. Rizzi, I. Rosset, and M. Roriz-Cruz, "Global epidemiology of dementia: Alzheimer's and vascular types," BioMed Research International, vol. 2014, Article ID 908915, 8 pages, 2014.

[5] J. T. O'Brien and A. Thomas, "Vascular dementia," Lancet, vol. 386, no. 10004, pp. 1698-1706, 2015.

[6] C. Iadecola, "The pathobiology of vascular dementia," Neuron, vol. 80, no. 4, pp. 844-866, 2013.

[7] R. N. Kalaria, R. Akinyemi, and M. Ihara, "Stroke injury, cognitive impairment and vascular dementia," Biochimica et Biophysica Acta, vol. 1862, no. 5, pp. 915-925, 2016. 
[8] P. B. Gorelick, S. E. Counts, and D. Nyenhuis, "Vascular cognitive impairment and dementia," Biochimica et Biophysica Acta, vol. 1862, no. 5, pp. 860-868, 2016.

[9] M. K. Sun, "Potential therapeutics for vascular cognitive impairment and dementia," Current Neuropharmacology, vol. 16, no. 7, pp. 1036-1044, 2018.

[10] G. J. Biessels, "Diagnosis and treatment of vascular damage in dementia," BiochimBiophys Acta., vol. 1862, no. 5, pp. 869877, 2016.

[11] W. M. van der Flier, I. Skoog, J. A. Schneider et al., "Vascular cognitive impairment," Nature Reviews. Disease Primers, vol. 4, no. 1, p. 18003, 2018.

[12] L. Zeng, Y. Zou, L. Kong et al., "Can Chinese herbal medicine adjunctive therapy improve outcomes of senile vascular dementia? Systematic review with meta-analysis of clinical trials," Phytotherapy Research, vol. 29, no. 12, pp. 1843-1857, 2015.

[13] D. Chang, J. Liu, K. Bilinski et al., "Herbal medicine for the treatment of vascular dementia: an overview of scientific evidence," Evidence-based Complementary and Alternative Medicine, vol. 2016, Article ID 7293626, 15 pages, 2016.

[14] Y. Zhai, N. Tang, J. H. Li et al., "A randomized controlled clinical study of Wuzang Wenyang Huayu decoction and stasis capsules in the treatment of vascular dementia," Liaoning Journal of Traditional Chinese Medicine, vol. 44, no. 6, pp. 12121214, 2017.

[15] T. Nong, G. Lian, and Y. Yan, "Clinical observation on the treatment of 87 cases of arteriosclerotic vascular dementia with Wuzang Wenyang Huayu decoction and stasis decoction," Lishizhen Medicine and Materia Medica, vol. 26, no. 6, pp. 1421-1422, 2015.

[16] W. Nong, Z. Q. Wei, X. N. Mo, L. Wu, and N. Tang, "Wenyang Wuzang Huayu Tangyu Decoction inhibits cyclin-dependent kinase-5 overexpression and its effect on neurofibrillary tangles in SAMP8 mice," Chinese Journal of Experimental Traditional Chinese Medicine, vol. 25, no. 9, pp. 61-66, 2019.

[17] J. Ru, P. Li, J. Wang et al., "TCMSP: a database of systems pharmacology for drug discovery from herbal medicines," Journal of Cheminformatics, vol. 6, no. 1, 2014.

[18] A. Hamosh, A. F. Scott, J. S. Amberger, C. A. Bocchini, and V. A. McKusick, "Online Mendelian Inheritance in Man (OMIM), a knowledge base of human genes and genetic disorders," Nucleic Acids Research, vol. 33, no. Database issue, pp. D514-D517, 2005.

[19] G. Stelzer, R. Rosen, I. Plaschkes et al., "The GeneCards suite: from gene data mining to disease genome sequence Analyses," Current Protocols in Bioinformatics, vol. 54, no. 1, pp. 1-30, 2016.

[20] A. Weiland, Y. Wang, W. Wu et al., "Ferroptosis and its role in diverse brain diseases," Molecular Neurobiology, vol. 56, no. 7, pp. 4880-4893, 2019.

[21] B. R. Stockwell, J. P. Friedmann Angeli, H. Bayir et al., "Ferroptosis: a regulated cell death nexus linking metabolism, redox biology, and disease," Cell, vol. 171, no. 2, pp. 273-285, 2017.

[22] B. Chen, Z. Chen, M. Liu et al., "Inhibition of neuronal ferroptosis in the acute phase of intracerebral hemorrhage shows long-term cerebroprotective effects," Brain Research Bulletin, vol. 153, pp. 122-132, 2019.

[23] I. Alim, J. T. Caulfield, Y. Chen et al., "Selenium drives a transcriptional adaptive program to block ferroptosis and treat stroke," Cell, vol. 177, no. 5, pp. 1262-1279.e25, 2019.
[24] Y. Fang, S. Gao, X. Wang et al., "Programmed cell deaths and potential crosstalk with blood-brain barrier dysfunction after hemorrhagic stroke," Frontiers in Cellular Neuroscience, vol. 14, p. 68, 2020.

[25] J. Wan, H. Ren, and J. Wang, "Iron toxicity, lipid peroxidation and ferroptosis after intracerebral haemorrhage," Stroke and Vascular Neurology, vol. 4, no. 2, pp. 93-95, 2019.

[26] D. Szklarczyk, A. Franceschini, S. Wyder et al., "STRING v10:protein-protein interaction networks, integrated over the tree of life," Nucleic Acids Research, vol. 43, no. D1, pp. D447-D452, 2015.

[27] G. D. Bader and C. W. Hogue, "An automated method for finding molecular complexes in large protein interaction networks," BMC Bioinformatics, vol. 4, no. 1, p. 2, 2003.

[28] D. W. Huang, B. T. Sherman, and R. A. Lempicki, "Systematic and integrative analysis of large gene lists using DAVID bioinformatics resources," Nature Protocols, vol. 4, no. 1, pp. 44-57, 2009.

[29] S. Tariq and P. A. Barber, "Dementia risk and prevention by targeting modifiable vascular risk factors," Journal of Neurochemistry, vol. 144, no. 5, pp. 565-581, 2018.

[30] M. Grieco, A. Giorgi, M. C. Gentile et al., "Glucagon-like peptide-1: a focus on neurodegenerative diseases," Frontiers in Neuroscience, vol. 13, p. 1112, 2019.

[31] A. D. Korczyn, V. Vakhapova, and L. T. Grinberg, "Vascular dementia," Journal of the Neurological Sciences, vol. 322, no. 1-2, pp. 2-10, 2012.

[32] H. V. Vinters, C. Zarow, E. Borys et al., "Vascular dementia: clinicopathologic and genetic considerations," Neuropathology and Applied Neurobiology, vol. 44, no. 3, pp. 247-266, 2018.

[33] M. Dichgans and D. Leys, "Vascular cognitive impairment," Circulation Research, vol. 120, no. 3, pp. 573-591, 2017.

[34] A. Wallin, G. C. Román, M. Esiri et al., "Update on vascular cognitive impairment associated with subcortical small-vessel disease," Journal of Alzheimer's Disease, vol. 62, no. 3, pp. 1417-1441, 2018.

[35] E. Mori, "Impact of subcortical ischemic lesions on behavior and cognition," Annals of the New York Academy of Sciences, vol. 977, no. 1, pp. 141-148, 2002.

[36] S. Li, T. P. Fan, W. Jia, A. Lu, and W. Zhang, "Network pharmacology in traditional Chinese medicine," Evidence-based Complementary and Alternative Medicine, vol. 2014, 2 pages, 2014.

[37] F. Forette, M. L. Seux, J. A. Staessen et al., "Prevention of dementia in randomised double-blind placebo-controlled Systolic Hypertension in Europe (Syst-Eur) trial," Lancet, vol. 352, no. 9137, pp. 1347-1351, 1998.

[38] X. Yu and T. Kensler, "Nrf2 as a target for cancer chemoprevention," Mutation Research, vol. 591, no. 1-2, pp. 93-102, 2005.

[39] F. A. al-Omar, M. N. Nagi, M. N. Abdulgadir, K. S. al Joni, and A. A. al-Majed, "Immediate and delayed treatments with curcumin Prevents forebrain ischemia-induced neuronal damage and oxidative insult in the rat hippocampus," Neurochemical Research, vol. 31, no. 5, pp. 611-618, 2006.

[40] R. M. Adibhatla and J. F. Hatcher, "Role of lipids in brain injury and diseases," Future Lipidology, vol. 2, no. 4, pp. 403422, 2007.

[41] R. M. Adibhatla and J. F. Hatcher, "Altered lipid metabolism in brain injury and disorders," Sub-Cellular Biochemistry, vol. 49, pp. 241-268, 2008. 
[42] R. M. Adibhatla, R. Dempsy, and J. F. Hatcher, "Integration of cytokine biology and lipid metabolism in stroke," Frontiers in Bioscience, vol. 13, no. 13, pp. 1250-1270, 2008.

[43] R. M. Adibhatla, J. F. Hatcher, and R. J. Dempsey, "Lipids and lipidomics in brain injury and diseases," The AAPS Journal, vol. 8, no. 2, pp. E314-E321, 2006.

[44] A. Lewén, P. Matz, and P. H. Chan, "Free radical pathways in CNS injury," Journal of Neurotrauma, vol. 17, no. 10, pp. 871890, 2000.

[45] R. M. Adibhatla and J. F. Hatcher, "Lipid oxidation and peroxidation in CNS health and disease: from molecular mechanisms to therapeutic opportunities," Antioxidants \& Redox Signaling, vol. 12, no. 1, pp. 125-169, 2010.

[46] X. L. Wang, "The neuroprotective effect of Nrf2 in central nervous system diseases," Journal of Medical Postgraduates, vol. 24, no. 7, pp. 754-757, 2011.

[47] R. Dringen, "Metabolism and functions of glutathione in brain," Progress in Neurobiology, vol. 62, no. 6, pp. 649-671, 2000.

[48] A. Y. Shih, P. Li, and T. H. Murphy, "A small-moleculeinducible Nrf2-mediated antioxidant response provides effective prophylaxis against cerebral Ischemia In vivo," The Journal of Neuroscience, vol. 25, no. 44, pp. 10321-10335, 2005.

[49] J. Zhao, N. Kobori, J. Aronowski, and P. K. Dash, "Sulforaphane reduces infarct volume following focal cerebral ischemia in rodents," Neuroscience Letters, vol. 393, no. 2-3, pp. 108-112, 2006. 